\title{
Estimating intra-axonal axial diffusivity in the presence of fibre orientation dispersion
}

\author{
Amy FD Howard ${ }^{\mathrm{a}}$, Frederik J Lange ${ }^{\mathrm{a}}$, Jeroen Mollink ${ }^{\mathrm{a}, \mathrm{b}}$, Michiel Cottaar ${ }^{\mathrm{a}}$, Mark Drakesmith ${ }^{\mathrm{c}, \mathrm{d}}$,

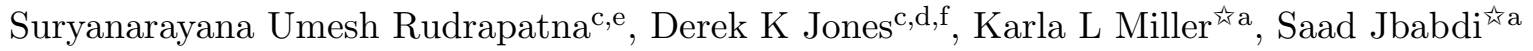 \\ ${ }^{a}$ FMRIB Centre, Wellcome Centre for Integrative Neuroimaging, Nuffield Department of Clinical Neurosciences, \\ University of Oxford, Oxford, United Kingdom \\ ${ }^{b}$ Department of Anatomy, Donders Institute for Brain, Cognition and Behaviour, Radboud University Medical \\ Centre (Radboudumc), Nijmegen, Netherlands \\ ${ }^{c}$ Cardiff University Brain Research Imaging Centre, Cardiff University, Cardiff, United Kingdom \\ ${ }^{d}$ Neuroscience and Mental Health Research Institute, Cardiff University, Cardiff, United Kingdom \\ ${ }^{e}$ Philips Innovation Campus, Bangalore, India \\ ${ }^{f}$ Mary MacKillop Institute for Health Research, Faculty of Health Sciences, Australian Catholic University, \\ Melbourne, Victoria, Australia
}

\begin{abstract}
By analysing the diffusion MRI signal, we can infer information about the microscopic structure of the brain. Two parameters of interest - the intra-axonal axial diffusivity and fibre orientation dispersion - are potential biomarkers for very different aspects of the white matter microstructure, yet they are difficult to disentangle. The parameters covary such that, if one is not accurately accounted for, the other will be biased. In this work we use high b-value data to isolate the signal from the intra-axonal compartment and resolve any degeneracies with the extra-axonal compartment. In the high b-value regime, we then use a model of dispersed sticks to estimate the intra-axonal axial diffusivity and fibre orientation distribution on a voxelwise basis. Our results in in vivo, human data show an intra-axonal axial diffusivity of $\sim 2.3-3 \mu \mathrm{m}^{2} / \mathrm{ms}$, where $3 \mu \mathrm{m}^{2} / \mathrm{ms}$ is the diffusivity of free water at $37^{\circ} \mathrm{C}$. The intra-axonal axial diffusivity is seen to vary considerably across the white matter. For example, in the corpus callosum we find high values in the genu and splenium, and lower values in the midbody. Furthermore, the axial diffusivity and orientation dispersion appear negatively correlated, behaviour which we show is consistent with the presence of fibre undulations but not consistent with a degeneracy between fanning fibres and axial diffusivity. Finally, we demonstrate that the parameter maps output from Neurite Orientation Dispersion and Density Imaging (NODDI) change substantially when the assumed axial diffusivity was increased from 1.7 to 2.5 or $3 \mu \mathrm{m}^{2} / \mathrm{ms}$.
\end{abstract}

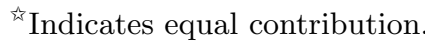




\section{Introduction}

By analysing the diffusion MRI signal, we can infer information about the tissue microstructure to reveal the structural connectivity of the human brain [1], or characterise cellular changes in the myelo- or cyto-architecture [2]. Such inference, however, requires accurate biophysical modelling of the tissue microstructure and accurate knowledge of the resultant diffusion characteristics [3, 4]. Furthermore, the model must be designed to consider the specific microstructural features which influence the diffusion signal.

Two parameters of interest are the intra-axonal axial diffusivity and the fibre orientation dispersion. The intra-axonal axial diffusivity describes the diffusion of water molecules inside the axon as they travel along the primary axis or orientation. Knowledge of the axial diffusivity often underpins our ability to accurately model the diffusion signal. The fibre orientation dispersion describes the extent of fibre fanning or coherence within a voxel. As a measure of white matter organisation, the orientation dispersion is important when determining structural connectivity and is a biomarker of brain development, ageing and various neurological diseases [5]. However, using typical data $\left(\sim b \leq 3 \mathrm{~ms} / \mu^{2}\right)$ acquired in the long diffusion time limit, it is difficult to simultaneously estimate the intra-axonal axial diffusivity and orientation dispersion for two reasons.

Firstly, when modelling the intra-axonal compartment, both the intra-axonal axial diffusivity and fibre orientation dispersion must be explicitly co-modelled otherwise the parameter estimates will be biased. This is because the intra-axonal axial diffusivity and fibre orientation dispersion covary such that an inaccurate axial diffusivity (if either assumed or estimated from the data) leads to a misestimation of orientation dispersion, and vice versa.

Secondly, we generally receive signal from both intra- and extra-axonal water and therefore need to co-model the diffusion properties of these compartments. Here there exists two degeneracies [6]: i) between the axial diffusivity of the intra- and extra-axonal compartments, and ii) between the orientation dispersion and the diffusion characteristics of the hindered extra-axonal compartment. For example, in the long diffusion time limit, an apparent radial diffusivity could be attributed to either the diffusion of water within the extra-axonal space, or that within a disperse fibre bundle.

To address these degeneracies, a common approach is to fix the diffusivities and estimate the fibre orientation dispersion. In constrained spherical deconvolution [7], the diffusion characteristics of the combined intra- and extra-axonal compartment (as described by the fibre response function) are typically first defined from the 'most likely' single-fibre voxels, and then assumed constant across the tissue to estimate the fibre orientation distribution. Neurite orientation dispersion and density imaging (NODDI) [5] takes an alternative approach, fixing the intra-axonal axial diffusivity to a global, predefined value (typically $1.7 \mathrm{~m}^{2} / \mathrm{ms}$ ). The axial diffusivity of the extra-axonal compartment is assumed equal to that of the intra-axonal compartment, and the extra-axonal radial diffusivity is defined by a tortuosity model that describes the hindrance of water in the extra-axonal space. Though these strong modelling assumptions make the problem tractable, numerous studies challenge their accuracy $[8,9,10,11,12]$ where, if the assumed diffusivities are inaccurate, the estimation of the remaining model parameters will be biased.

In this work we aim to estimate the intra-axonal axial diffusivity by i) only considering signal from the intra-axonal compartment - this is achieved through high b-value, in vivo human data - and ii) 
explicitly modelling both the orientation dispersion and axial diffusivity on a voxelwise basis. High b-value is crucial because it eliminates the higher-diffusivity extra-axonal water such that only the intra-axonal compartment contributes signal [13, 14, 15, 4, 16, 17, 18], thus overcoming the above described degeneracies and allowing the simultaneous estimation of intra-axonal axial diffusivity and fibre orientation dispersion. We first demonstrate in simulated data the effect of getting some of the model assumptions wrong. For example, as the model assumes symmetric fibre dispersion, we examine how the parameter estimates behave when the model is applied to simulated data with asymmetric dispersion. The model was then applied to high b-value data from 32 human subjects scanned with ultra-strong gradients. By estimating the axial diffusivity per voxel - rather than fitting to the average signal across many voxels - we demonstrate how the axial diffusivity appears to vary across the brain. Furthermore, we find a negative correlation between the axial diffusivity and orientation dispersion and demonstrate how fibre undulations may explain this behaviour. Finally, we explore how changing the assumed axial diffusivity in the NODDI model biases the remaining estimated parameters.

\section{Theory}

The diffusion signal $S$ can be considered the summation of signal contributions $S_{i=1 . . N}$ from $N$ compartments, weighted by their respective signal fractions $f_{i}$, where

$$
S=\sum_{i}^{N} f_{i} S_{i} .
$$

For example, in multi-compartment models, a proportion of the signal may be attributed to, interalia, the intra-axonal, extra-axonal or CSF compartments respectively. Note here that the signal fractions $f_{i}$ do not necessarily equate to the volume fractions of the relative compartments [19].

At high b-values, the signal contribution from compartments with highly mobile water become negligible $[13,14,15,4,16,17,18]$ and the diffusion signal can be reasonably attributed to arising solely from the intra-axonal compartment,

$$
S \approx f_{i n} S_{i n}
$$

\subsection{The dispersed stick model}

The dispersed stick model is specifically designed for high b-value data where the entirety of the diffusion signal can be attributed to the intra-axonal compartment $[13,14,15,4,16,17,18]$. The model is graphically described in Figure 1. In the model, the diffusion signal along gradient direction $\boldsymbol{g}$ is given by the convolution of the fibre orientation distribution, which is assumed to be a Watson distribution $[20,5]$, and a fibre response function for stick-like fibres with Gaussian axial diffusion, and no radial diffusion:

$$
S \approx f_{i n} S_{0} \frac{1}{C_{W}} \int_{S^{2}} \exp \left[\kappa\left(\boldsymbol{\mu}^{\top} \boldsymbol{x}\right)^{2}\right] \cdot \exp \left[-b d_{\|}\left(\boldsymbol{g}^{\top} \boldsymbol{x}\right)^{2}\right] \mathrm{d} S,
$$


where the integrand is over $\boldsymbol{x} \in S^{2} ; f_{\text {in }}$ is the signal fraction of the intra-axonal compartment; $d_{\|}$ is the intra-axonal axial diffusivity; $\boldsymbol{x}$, a unit vector on the sphere $S ; b$, the b-value and $S_{0}$ is the non-diffusion weighted signal $S(b=0)$. Here the fibre orientation distribution is described by a Watson distribution $[20,5]$,

$$
\frac{1}{C_{W}} \exp \left[\kappa\left(\boldsymbol{\mu}^{\top} \boldsymbol{x}\right)^{2}\right]
$$

with orientation $\boldsymbol{\mu}$, dispersion $\kappa$ and normalising constant $C_{W}$, where,

$$
C_{W}=\int_{S^{2}} \exp \left[\kappa\left(\boldsymbol{\mu}^{\top} \boldsymbol{x}\right)^{2}\right] \mathrm{d} S=\int_{S^{2}} \exp \left[\boldsymbol{x}^{\top}\left[\kappa \boldsymbol{\mu} \boldsymbol{\mu}^{\top}\right] \boldsymbol{x}\right] \mathrm{d} S=4 \pi_{1} F_{1}\left(1 / 2 ; 3 / 2 ; \kappa \boldsymbol{\mu} \boldsymbol{\mu}^{\top}\right) .
$$

${ }_{1} F_{1}(\alpha ; \beta ; \boldsymbol{X})$ is the confluent hypergeometric function of the first kind with a matrix argument $\boldsymbol{X}$.

The orientation dispersion index is defined as $\mathrm{ODI}=2 / \pi \arctan (1 / \kappa)[5]$ and ranges from 0 to 1 , representing perfectly aligned and isotropic fibre distributions respectively. To aid comparison with previous literature, Appendix A shows how the ODI relates to the fanning angle in degrees.

The model thus has five free parameters: the axial diffusivity, $d_{\|}$; the orientation dispersion index, ODI; $\theta$ and $\phi$ which describe the primary fibre orientation of the Watson distribution, $\boldsymbol{\mu}(\theta, \phi)$ and $F=f_{i n} S_{0}$, the scaling parameter. To solve for all parameters simultaneously and without parameter degeneracy, the dispersed stick model requires data with three b-values or shells, each with negligible contributions from the extra-axonal compartment i.e. $b>6,000 \mathrm{~ms} / \mathrm{um}^{2}[21]$.

Note, the intra-axonal signal fraction with respect to the non-diffusion weighted signal, $f_{i n}$, can be approximated as $F / S_{0}$. As both the intra- and extra-axonal compartment contribute to the $S_{0}$ signal, this approximation assumes that the intra- and extra-axonal water has the same T2 properties. As this is unlikely true [22], the approximate $f_{\text {in }}$ should be interpreted with caution.

The model is based on four main assumptions:

1. The fibre orientation distribution is accurately described by a Watson distribution.

2. Water experiences axons to be like sticks i.e. with no measurable radial diffusion.

3. There is a negligible contribution from extra-axonal water.

4. The voxel contains a single dispersed fibre population.

The latter is enforced for simplicity but could potentially be relaxed in future work. Furthermore, though there is likely a range of diffusivities within a voxel - where axons of different morphology (e.g. caliber or undulation) may have slightly different axial diffusivities - this analysis assumes that each voxel can be described by a single, 'characteristic' diffusivity. Finally, as the exchange time has been previously estimated to be considerably larger than typical diffusion times (seconds [23] versus milliseconds) we assume the axons to be impermeable with no slow exchange.

These assumptions require high b-value data which - to efficiently acquire data with high signalto-noise ratio - are typically acquired using ultra-strong gradient sets. As these gradient sets are available on only a few human scanners, the assumptions of the model likely limit its widespread application. However, the assumptions do enable the simultaneous estimation of the fibre dispersion and intra-axonal axial diffusion on a voxelwise basis, something that is difficult to do when also accounting for the extra-axonal space. Thus, the model may provide useful insights into quantifying 
a) The dispersed stick model
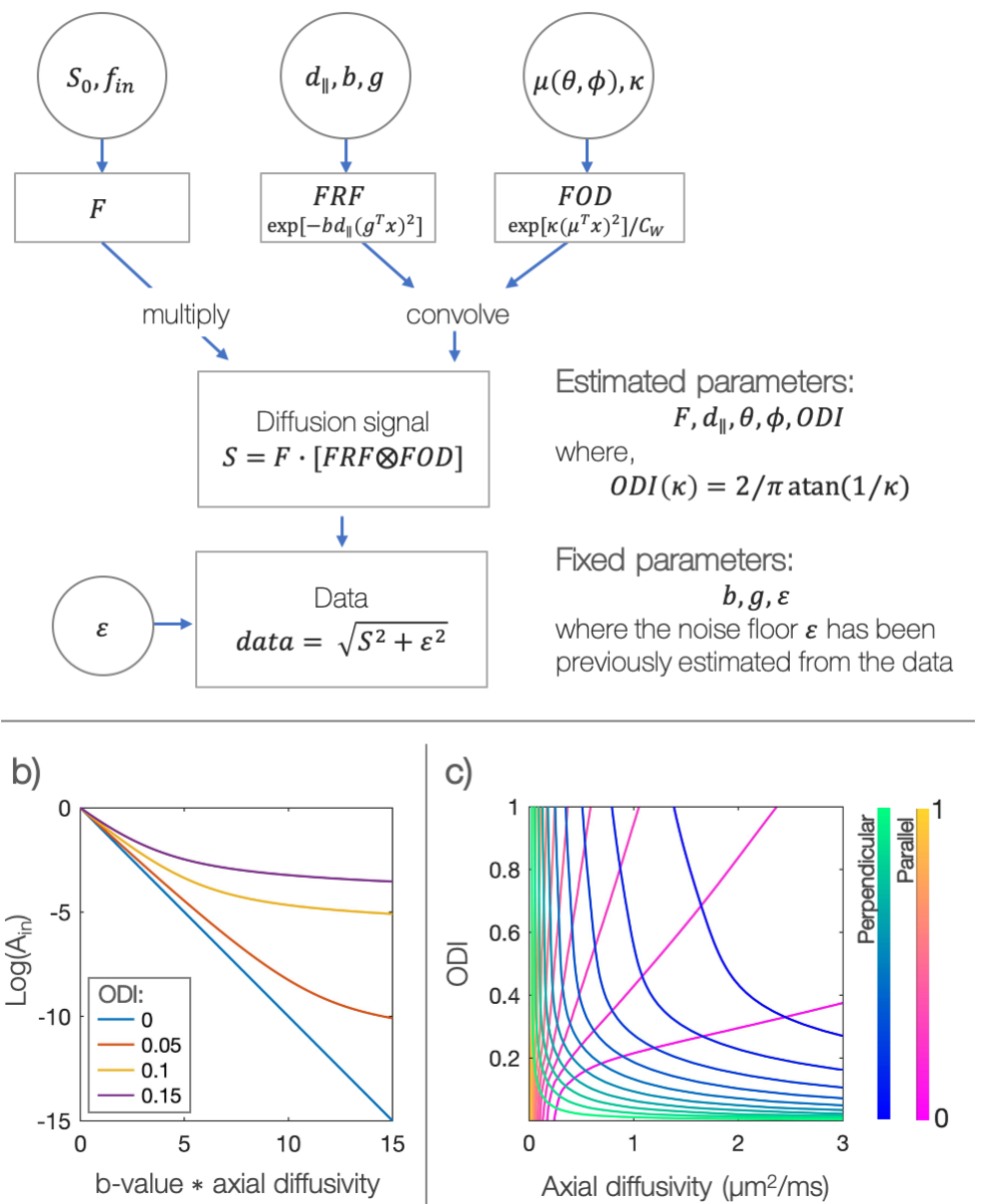

Figure 1: The dispersed stick model. a) In the generative model, the diffusion signal is given by the convolution of the fibre response function $(F R F)$ and the fibre orientation distribution, $(F O D)$, which is then scaled by the intra-axonal signal fraction $f_{i n}$ and the non-diffusion-weighted signal $S_{0}$. For high b-value data, the $F R F$ is solely attributed to the intra-axonal compartment with axial diffusivity $d_{\|}$. The $F O D$ is described as a Watson distribution with primary orientation $\boldsymbol{\mu}(\theta, \phi)$ and dispersion $\kappa$. Finally, the signal is combined quadratically with the noise floor $\epsilon$ which is first estimated from the data, and then assumed constant across the brain. b) In the presence of fibre orientation dispersion, the diffusion signal appears to vary with b-value. Thus, the orientation dispersion and axial diffusion should be estimated jointly. c) Equation 3 can be solved analytically for gradient directions $\boldsymbol{g}$ both perpendicular (blue-green) and parallel (pink-yellow) to the fibre $\boldsymbol{\mu}$. When analysed separately, each gradient orientation produces a degenerate solution (each line shows combinations of ODI and axial diffusivity which produce the same diffusion signal), but when combined, provide a unique solution (where the lines intersect). 
the value of, and variations in, intra-axonal axial diffusivity across the brain. This information can then influence the development of future, more accurate, diffusion models.

Figure $1 \mathrm{~b}$ shows the diffusion attenuation along the fibre $(\boldsymbol{g}=\boldsymbol{\mu})$ as a function of $b * d_{\|}$for various levels of dispersion. Here we assume the signal arises solely from the intra-axonal compartment, $F=S_{0}$. With even a small amount of fibre dispersion within an imaging voxel, the intra-axonal axial diffusivity appears to vary with b-value. Note, an ODI of 0.05 or 0.1 relates to a dispersion angle (containing $50 \%$ of the fibres) of only $15^{\circ}$ or $22^{\circ}$ respectively (see Appendix A). If dispersion is ignored, this apparent deviation from Gaussian diffusion (a.k.a. non-zero kurtosis) could be erroneously assigned to a complex diffusion micro-environment other than fibre dispersion (a microstructure properties viewpoint), or it could bias the estimation of the axial diffusivity (a diffusion properties viewpoint). Thus Figure $1 \mathrm{~b}$ highlights the importance of accurately determining the intra-axonal axial diffusivity on a voxelwise basis.

\subsection{The analytic form}

The dispersed stick model has an analytic form. The integral in Equation 3 can be written as the confluent hypergeometric function of the first kind with matrix argument $X=\kappa \boldsymbol{\mu} \boldsymbol{\mu}^{\top}-b d_{\|} \boldsymbol{g g} \boldsymbol{g}^{\top}$, where

$$
\begin{aligned}
\int_{S^{2}} \exp \left[\kappa\left(\boldsymbol{\mu}^{\top} \boldsymbol{x}\right)^{2}\right] \cdot \exp \left[-b d_{\|}\left(\boldsymbol{g}^{\top} \boldsymbol{x}\right)^{2}\right] \mathrm{d} S=\int_{S^{2}} \exp \left[\boldsymbol{x}^{\top}\left(\kappa \boldsymbol{\mu} \boldsymbol{\mu}^{\top}-b d_{\|} \boldsymbol{g} \boldsymbol{g}^{\top}\right) \boldsymbol{x}\right] \mathrm{d} S \\
=4 \pi_{1} F_{1}\left(1 / 2 ; 3 / 2 ; \kappa \boldsymbol{\mu} \boldsymbol{\mu}^{\top}-b d_{\|} \boldsymbol{g g}^{\top}\right)
\end{aligned}
$$

Thus, the diffusion signal attenuation is given by,

$$
A_{\text {in }}=\frac{{ }_{1} F_{1}\left(1 / 2 ; 3 / 2 ; \kappa \boldsymbol{\mu} \boldsymbol{\mu}^{\top}-b d_{\|} \boldsymbol{g g}^{\top}\right)}{{ }_{1} F_{1}\left(1 / 2 ; 3 / 2 ; \kappa \boldsymbol{\mu} \boldsymbol{\mu}^{\top}\right)},
$$

and the diffusion signal by,

$$
S \approx f_{i n} S_{0} \frac{{ }_{1} F_{1}\left(1 / 2 ; 3 / 2 ; \kappa \boldsymbol{\mu} \boldsymbol{\mu}^{\top}-b d_{\|} \boldsymbol{g g}^{\top}\right)}{{ }_{1} F_{1}\left(1 / 2 ; 3 / 2 ; \kappa \boldsymbol{\mu} \boldsymbol{\mu}^{\top}\right)} .
$$

Two examples of the analytic form are shown in Figure 1c: when the gradient is parallel and perpendicular to the fibre. Individual lines in Figure 1c represent all pairs of ODI and $d_{\|}$that are consistent with a given diffusion signal. We see how the combined analysis of $\geq 2$ unique gradient directions (which don't have the same angle to the fibre orientation $\boldsymbol{\mu}$ ) provides a unique solution for both the within-voxel fibre dispersion and axial diffusivity, i.e. each blue-green line intersects a pink-yellow line at exactly one point.

\section{Methods}

\subsection{Model optimisation}

During optimisation, the five parameters of the model were bounded such that $d_{\|} \in[0,4] \mu \mathrm{m}^{2} / \mathrm{ms}$, ODI $\in[0,1], F>0$, as well as $\theta$ and $\phi$ being unbounded. Note that the absolute limit of the 
axial diffusivity in pure water at $37^{\circ} \mathrm{C}$ is $\sim 3-3.1 \mu \mathrm{m}^{2} / \mathrm{ms}$, far less than the upper limit of $4 \mathrm{\mu m}^{2} / \mathrm{ms}$ used here. To reduce computation time, the in vivo data were optimised using the analytic solution and graphical processing units (GPUs) using FSL's CUDA Diffusion Modelling Toolbox, cuDIMOT [24]. The model was optimised using Markov Chain Monte Carlo (MCMC) which afforded estimation of each parameter's distribution - and Rician noise modelling, both of which are internal functions of cuDIMOT [24].

The dispersed stick model was applied to multi-shell in vivo human data acquired at b-values of $7.75,11.6$ and $15.5 \mathrm{~ms} / \mathrm{\mu m}^{2}$ (data described in full below, c.f. 3.3.1 High b-value data). Upon inspection, data acquired parallel to the primary fibre orientation approached the rectified noise floor [25] which was subsequently accounted for in the model (Figure 2):

$$
\text { data }=\sqrt{S^{2}+\epsilon^{2}}
$$

Prior to model optimisation, the subject-specific noise floor $\epsilon$ was estimated directly from the data. Here the noise floor was taken to be the mean signal from voxels located in the ventricles for data with 30 gradient directions and $b=15.5 \mathrm{~ms} / \mathrm{\mu m}^{2}$.

\subsection{Simulations: model violations}

Simulated data were used to examine how deviating from the assumptions 1-4 of section 2.2.1 in turn affected our two main parameters of interest: the axial diffusivity and ODI. Using generative models, data were simulated for conditions which violate our assumptions - asymmetric dispersion, non-zero radial diffusion, extra-axonal contributions and two fibre populations - after which the model was fitted to these data. Each of these simulated violations of our assumptions is described in detail below.

Multi-shell data were simulated using the same b-values and gradient directions as the in vivo data described below (c.f. In vivo data): 30 gradient directions each for $b=7.75,11.6,15.5 \mathrm{~ms} / \mathrm{um}^{2}$ and with $S_{0}=125$. No noise was added to the data. During optimisation the fibre orientation $\boldsymbol{\mu}(\theta=0, \phi=0)$ was fixed and the model optimised to estimate the axial diffusivity and ODI. When the simulated data contained contributions from the extra-axonal space, the scaling parameter $F=f_{\text {in }} * S_{0}$ was also fitted. For all other simulated data (i.e. with asymmetric dispersion, nonzero radial diffusion and two fibre populations), the scaling parameter was fixed to the ground truth value $F=0.5 * S_{0}$. The ground truth values for axial diffusivity and ODI were $2.2 \mathrm{\mu m}^{2} / \mathrm{ms}$ and 0.1 respectively, where the latter is equivalent to a $50 \%$ opening angle of $22^{\circ}$ (Appendix A). In each case, the model was fitted assuming stick-like fibres described by a Watson distribution with signal contributions arising from only the intra-axonal compartment. The model was optimised using the Metropolis Hastings method [26] where the mean of each parameter distribution is reported.

\subsubsection{Violating assumption 1: asymmetric fibre orientation distribution}

The model assumes a Watson fibre orientation distribution characterised by symmetric dispersion about a primary fibre orientation $[20,5]$. However, the true distribution is likely much more complex. Here we simulated data for a fibre orientation distribution described by a Bingham 
distribution to depict asymmetric fibre dispersion [27, 28, 29]. The Bingham distribution has two axes of dispersion about the primary fibre orientation $\boldsymbol{\mu}$, with dispersion constants $\kappa_{1}$ and $\kappa_{2}$. When $\kappa_{1}=\kappa_{2}$, the dispersion is symmetric and the Bingham is again equivalent to the Watson distribution. A Bingham distribution can therefore describe, for example, 'sheet-like' fibre distributions which are tightly aligned along one axis, but highly disperse along a second. Data were simulated for Bingham distributions with varying degrees of asymmetry, $\mathrm{ODI}_{1} / \mathrm{ODI}_{2}=$ $[1,3,9]$, whilst keeping the mean dispersion constant.

\subsubsection{Violating assumption 2: non-zero radial diffusivity}

The model assumes stick-like axons with water diffusion purely along the fibre and no radial diffusivity. Here data were simulated for fibres with non-zero radial diffusivity, where the diffusion profile - characterised by an axially symmetric diffusion tensor with $d_{\|}=2 \mathrm{~mm}^{2} / \mathrm{ms}$ and $d_{\perp}=$ $0,0.05,0.01,0.02 \mathrm{~mm}^{2} / \mathrm{ms}$ - was convolved with the disperse fibre orientation distribution.

\subsubsection{Violating assumption 3: extra-axonal compartment}

The model assumes that, by working in the high b-value regime, the diffusion signal from the extra-axonal space is negligible and thus the signal can be attributed solely to the intra-axonal compartment. Here, data were simulated for a two compartment model: an intra-axonal compartment described by stick-like diffusion, and an extra-axonal compartment described by an axiallysymmetric diffusion tensor. In both compartments, the diffusion profile was convolved with the disperse fibre distribution. As in the NODDI model [5], the extra-axonal axial diffusivity was equal to the intra-axonal axial diffusivity, $d_{e x, \|}=d_{i n, \|}=d_{\|}$, whilst the extra-axonal radial diffusivity was linked to the volume fraction of the extra-axonal compartment where $d_{e x, \perp}=d_{\|}\left(1-f_{i n}\right)$. The intra- and extra-axonal volume fractions sum to one i.e. $f_{i n}+f_{e x}=1$.

\subsubsection{Violating assumption 4: multiple fibre populations}

The model assumes that there is a single fibre population in each voxel, though many of the white matter voxels contain more than one fibre population. Here data were simulated for a voxel with two fibre populations of equal weighting or signal fraction. The average fibre orientation was held constant whilst the angle between the fibres was varied, $\gamma=0-60^{\circ}$. Each fibre population had a ground truth ODI of 0.1 .

\subsection{Simulations: undulations}

As we will show in Figure 7 (c.f 4.2 High b-value, in vivo data), for voxels with low dispersion, there is a negative relationship between intra-axonal axial diffusivity and fibre orientation dispersion. Given that a degeneracy between these parameters would result in the opposite relationship (positive correlation), this cannot be explained due to fitting. Therefore, we questioned whether there was some feature of the data that was unaccounted for in the model, but which resulted in a signal that our model could only describe through a coupling of low dispersion with high axial diffusivity (and vice versa). 
One possibility is if, rather than fanned sticks, fibre undulations lead to apparent dispersion [30, 31, 32, 33, 34]. Though both fibre fanning and undulations are arguably a form of microscopic dispersion, fibre fanning is an effect of pooling over a number of axons and undulations are a feature of individual axons. Whilst both may contribute the total amount of dispersion within a voxel, they may affect the apparent axial diffusivity in different ways. Intuitively, undulations could reduce the apparent intra-axonal axial diffusivity as water diffusing along the primary fibre "axis" would experience hindrance due to interactions with the meandering membrane wall.

To explore how fibre undulations affect the estimated intra-axonal axial diffusivity and ODI, data were simulated for a simple 2D set-up in which the wavelength and amplitude of fibre undulations could be varied. The dispersed stick model was then fitted to these data. The diffusion MRI signal was simulated using a Monte-Carlo simulation of particle displacement according to Brownian motion. To imitate diffusion along an undulating fibre of zero-radius, the particle movements were confined to a single 2D line described by a sinusoid,

$$
f(z)=[A \cos (2 \pi z / \lambda), 0, z],
$$

where $f(z)$ defined the particle position when confined to a fibre oriented along the $z$ axis with curvature or undulations along $x ; A$ and $\lambda$ are the amplitude and wavelength of the undulations respectively.

For an incremental time step $t=0.1 \mathrm{~ms}$, each particle stepped tangentially to the curve, where the step size is randomly drawn from a Gaussian distribution $\mathcal{N}(0, \sqrt{2 \Delta t})$. A pair of diffusion sensitising gradients of strength $G$, duration $\delta$ and spacing (i.e. diffusion time) $\Delta$ were applied, during which each spin accrued phase according to its position. The diffusion signal was then calculated as the magnitude of the sum of signals from all particles. The gradient strength $G$ was dependent on the desired b-value, where

$$
G=\frac{\sqrt{(b /(\Delta-\delta / 3))}}{\gamma \delta} .
$$

The acquisition parameters $b, \Delta$, and $\delta$ were chosen to mimic those of the in vivo data used in this study.

\subsection{In vivo human data}

\subsubsection{High b-value data}

MRI data were acquired for 32 healthy participants at the Cardiff University Research Imaging Centre (CUBRIC). The study was approved by the Cardiff University School of Psychology Ethics Committee and written informed consent was obtained from all subjects. Participants were scanned on a 3 T Connectom scanner (Siemens Healthcare, Erlangen, Germany) with an ultra-strong 300 $\mathrm{mT} / \mathrm{m}$ gradient set $[35,36]$.

T1-weighted images were used to register each subject to standard space. T1-weighted images were acquired with a Magnetization-Prepared Rapid Acquisition Gradient Echo (MPRAGE) sequence and $1 \mathrm{~mm}$ isotropic resolution. The T1 images were bias corrected (FSL's FAST [37]) and nonlinearly registered to MNI standard space (FSL's FNIRT [38, 39]). 


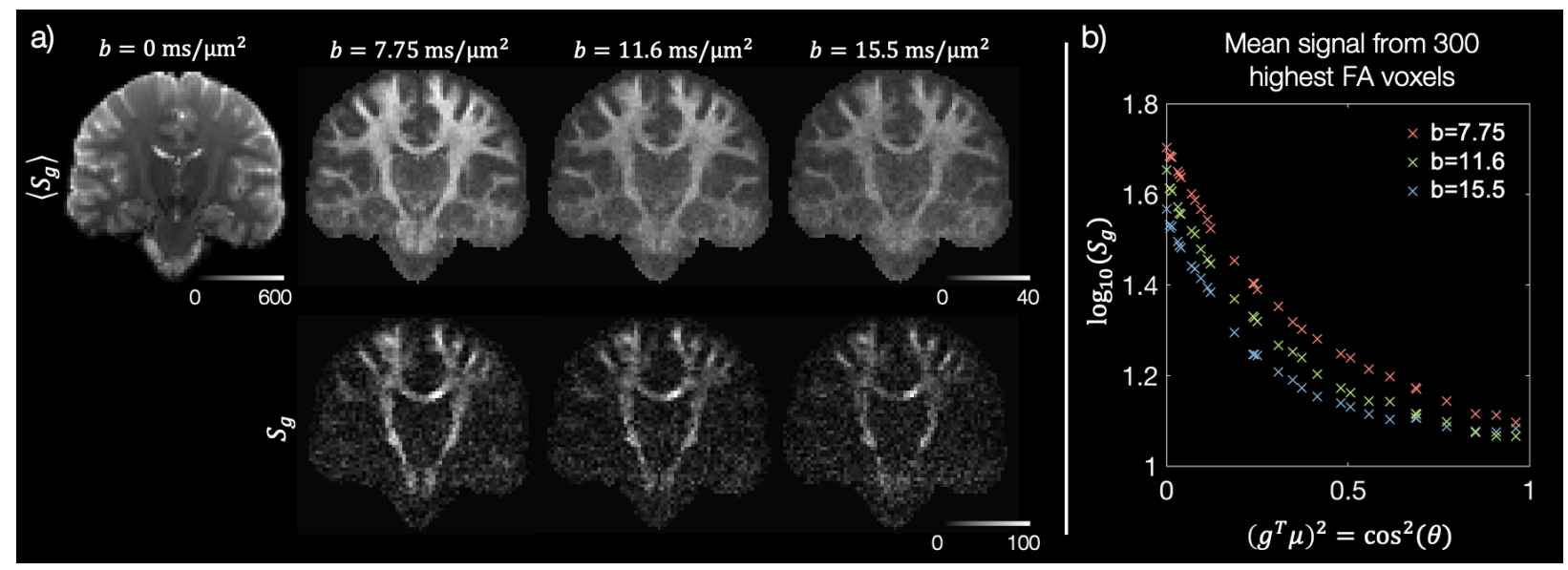

Figure 2: a) Example diffusion weighted images from a single subject. Top: the mean image across all gradient directions for $b=0 \mathrm{~ms} / \mathrm{\mu m}^{2}$ (12 volumes), $b=7.75,11.6$ and $15.5 \mathrm{~ms} / \mathrm{\mu m}^{2}$ (30 volumes each). Bottom: an example diffusion-weighted image. Here we see how, at very high b-values, we retain considerable signal in the tracts perpendicular to the gradient direction and high diffusion contrast. b) The mean signal intensity from the 300 highest FA voxels is plotted as a function of $\theta$, the angle between the fibres primary axis $\boldsymbol{\mu}$ and the gradient direction $\boldsymbol{g}$.

Diffusion-weighted data were acquired with a spin-echo Echo Planar Imaging (EPI) sequence (blipped-CAIPI) [40]: TR/TE $=3900 / 80 \mathrm{~ms}, F O V=220 \times 220 \mathrm{~mm}$, matrix $=110 \times 110,66$ slices, $2 \mathrm{~mm}$ isotropic voxels and Generalized Autocalibrating Partially Parallel Acquisition (GRAPPA) factor $=2$. Data were acquired at b-values of $7.75,11.6$ and $15.5 \mathrm{~ms} / \mathrm{\mu m}^{2}$ where $\delta / \Delta=7 / 42 \mathrm{~ms}$ for $b=11.6 \mathrm{~ms} / \mathrm{\mu m}^{2}$ and $\delta / \Delta=7 / 55 \mathrm{~ms}$ for $b=7.75,15.5 \mathrm{~ms} / \mathrm{\mu m}^{2} .30$ gradient directions per b-value. An additional 13 volumes were acquired with negligible diffusion weighting $\left(b \sim 0 \mathrm{~ms} / \mathrm{um}^{2}\right): 12$ were interspersed in the diffusion-weighted volumes to track subject motion and the 13th was acquired with reverse phase encoding for susceptibility distortion correction. Note, the $b \sim 0 \mathrm{~ms} / \mathrm{\mu m}^{2}$ data were not used in the fitting of the dispersed stick model.

The diffusion-weighted data were corrected for susceptibility and eddy current distortions, as well as subject motion (FSL's TOPUP [41, 42] and EDDY with outlier replacement [43, 44]). The dispersed stick model was optimised on the distortion corrected data in subject space, after which the results were warped to standard space and the mean map across subjects calculated.

Figure 2 shows example preprocessed images from the diffusion protocol. In Figure 2a we see how the images retain good signal and excellent diffusion contrast at very high b-values. Figure $2 \mathrm{~b}$ shows the mean diffusion signal for each b-value as a function of $\theta$, the angle between the fibre's primary axis $\boldsymbol{\mu}$ and the gradient direction $\boldsymbol{g}$. Here the 300 highest FA voxels were selected and reoriented to align with the $\mathrm{z}$ axis. Using linear interpolation, the diffusion signal was estimated for each voxel along consistent gradient directions. The mean signal across voxels for each gradient direction is shown. Here we see the signal from all three b-values plateau around $\log _{10}\left(S_{g}\right) \sim 1.08$, indicative of the signal attenuation parallel to the fibre reaching a noise floor of this magnitude. 


\subsubsection{Human Connectome Project data}

As we will show in section 4.2 Results: High b-value in vivo data, the dispersed stick model estimates an intra-axonal axial diffusivity in the range of $2.3-3 \mu \mathrm{m}^{2} / \mathrm{ms}$ with an average of $2.52 \mu \mathrm{m}^{2} / \mathrm{ms}$ in the white matter. This is considerably higher than that assumed by one of the most commonly used diffusion models NODDI. To estimate the neurite orientation dispersion and 'density', NODDI [5] assumes a predefined value of axial diffusivity which is typically $d_{\|}=1.7 \mu \mathrm{m}^{2} / \mathrm{ms}$. Due to the discrepancy between the assumed intra-axonal axial diffusivity of NODDI and the intra-axonal axial diffusivity estimates found in this study, we decided to explore how changing the assumed axial diffusivity affected the output of NODDI.

As NODDI models both the intra- and extra-axonal space, we were unable to use the high b-value data above, and so instead utilised preprocessed T1-weighted and diffusion-weighted data for the first 10 subjects of the WU-Minn Human Connectom Project (HCP); for details of the acquisition protocol and preprocessing pipeline, please see [45, 46, 47]. Briefly, the diffusion-weighted data included 90 gradient directions each at b-values of $b=1,2$ and $3 \mathrm{~ms} / \mathrm{um}^{2}$ and 18 interspersed volumes with negligible diffusion weighting. The distortion corrected diffusion-weighted data were linearly registered to each subjects T1-weighted structural scan (FSL's FLIRT [48, 49]), and the T1 non-linearly registered the MNI standard space (FSL's FNIRT [38, 39]). The NODDI model was applied to the diffusion data with an assumed axial diffusivity of either $d_{\|}=1.7,2.5$ or $3 \mu \mathrm{m}^{2} / \mathrm{ms}$.

\section{Results}

\subsection{Simulations: violating modelling assumptions}

Figure 3 explores our sensitivity to the modelling assumptions. In Figure 3a, data were simulated for a fibre orientation distribution described by a Bingham distribution with asymmetric dispersion along two axes $\left(\mathrm{ODI}_{1,2}\right)$. Here the 'ground truth' ODI represents the average dispersion across both axes which was kept constant at $\langle\mathrm{ODI}\rangle=0.1$. We see a negative relationship between the estimated axial diffusivity and ODI where for fibre populations with highly asymmetric dispersion, i.e. 'sheetlike' fibres, the orientation dispersion is underestimated and the axial diffusivity overestimated. For lower - and perhaps more realistic - levels of fibre asymmetry, the difference between the estimated and ground truth axial diffusivity and ODI is small.

In Figure 3b, axons are considered prolate tensors instead of sticks, with some diffusivity perpendicular to the fibre, $d_{\perp}$, here described by an axially-symmetric diffusion tensor. We see how violation of the stick-model substantially increases the estimated diffusivity, whilst the ODI is relatively unaffected.

In Figure 3c, both an intra-axonal and extra-axonal compartment contribute to the simulated diffusion signal. We see how when the signal fraction of the intra-axonal compartment is very large $f_{i n}=0.9$ (so that the extra-axonal compartment is small, $f_{\text {ex }}=1-f_{i n}$ ), contributions from the extra-axonal compartment increase the estimated axial diffusivity, whilst the ODI remains close to the ground truth value. Furthermore, there is a non-linear relationship between the estimated axial diffusivity and the intra-axonal volume fraction where, when $f_{i n}<0.9$, the overestimation of the axial diffusivity is reduced as $f_{\text {in }}$ decreases. These results can be understood if we consider 
two effects: i) the extra-axonal radial diffusivity is determined by the intra-axonal signal fraction $d_{e x, \perp}=d_{\|}\left(1-f_{\text {in }}\right)$ such that when $f_{\text {in }}$ is large, $d_{\|}$is small, and ii) at high b-value, the diffusion signal is sensitive to compartments where the diffusivity is small (whilst signal contributions from compartments with higher diffusivities are negligible). Thus, when $f_{i n}$ is large (and thus $d_{e x, \perp}$ small), our signal is sensitive to contributions from the extra-axonal space, but when $f_{\text {in }}$ and thus $d_{e x, \perp}$ decreases, the contributions from the extra-axonal compartment diminish. Notably, in Figure $3.3 \mathrm{c}$, when $0.5<f_{\text {in }}<0.7$ (i.e. more typically observed intra-axonal signal fractions [3]), the overestimation of the intra-axonal axial is relatively small $\left(d_{\|}=2.2-2.3 \mu^{2} / \mathrm{ms}\right)$.

Finally, Figure 3d shows how the estimated ODI is increased from the ground truth and the axial diffusivity reduced when data were simulated for two fibre populations of equal weighting. Here the two fibre populations have a fixed average orientation and variable opening angle $0 \leq \gamma \leq 60$. Note that these details would likely differ when investigating more complex multiple fibre populations where, for example, the fibre populations are unequally weighted or where each has a unique value of axial diffusivity.

\subsection{Model estimates in high b-value, in vivo data}

The dispersed stick model was applied to multi-shell, high b-value data from 32 subjects. Figure 4 shows maps of the mean intra-axonal axial diffusivity, ODI and intra-axonal signal fraction across all subjects. In the vast majority of voxels across the brain, the intra-axonal axial diffusivity lies between $\sim 2.3 \mathrm{\mu m}^{2} / \mathrm{ms}$ and $3 \mathrm{\mu m}^{2} / \mathrm{ms}$ with generally higher axial diffusivity in the white matter than the grey matter $\left(\left\langle d_{\|}\right\rangle_{w m}=2.52 \mu \mathrm{m}^{2} / \mathrm{ms}\right.$ and $\left\langle d_{\|}\right\rangle_{g m}=2.39 \mathrm{\mu m}^{2} / \mathrm{ms}$ respectively $)$. Note, the dispersed stick model may not be a good representation of the tissue structure of the grey matter which comprises cell bodies, dendrites and axons. Though it is typically assumed that stick like diffusion likely represents that from both axons and dendrites where, in the grey matter, the intraaxonal axial diffusivity could better interpreted as an intra-neurite axial diffusivity, recent work by Lampinen et al. [50] challenges the assumption of stick-like dendrites and we should interpret these grey matter results with caution. Nonetheless, the maps of ODI and intra-axonal signal fraction are broadly as we would expect: the ODI is low in the white matter and high in the grey matter, whilst the intra-axonal signal fraction is high in the white matter and low in the grey matter. Note, the signal fraction map is somewhat noisy in low $f_{i n}$ regions of the grey matter. Finally, the maps in Figure 4 demonstrate left-right symmetry and spatial smoothness, matching symmetric and smoothly changing anatomy. From here on in, we focus on the results in the white matter where the orientation dispersion is low and the model likely more representative of the underlying tissue.

\subsubsection{Variations across the white matter}

In Figure 4 , the axial diffusivity is particularly high $\left(d_{\|} \geq 2.6 \mu \mathrm{m}^{2} / \mathrm{ms}\right)$ and ODI particularly low $(\mathrm{ODI}<0.1)$ in regions of the corpus callosum, forceps major, forceps minor, fornix, anterior thalamic radiation, corticospinal tract, optic radiation, inferior fronto-occipital fasciculus, portions of the superior longitudinal fasciculus, as well as regions of the brain stem including the superior cerebellar peduncle, medial lemniscus and pontine crossing tract. Interestingly, the highest values of axial diffusivity $\left(d_{\|} \geq 2.9 \mathrm{\mu m}^{2} / \mathrm{ms}\right)$ were found mostly in the genu of the corpus callosum, though 
a) Asymmetric dispersion

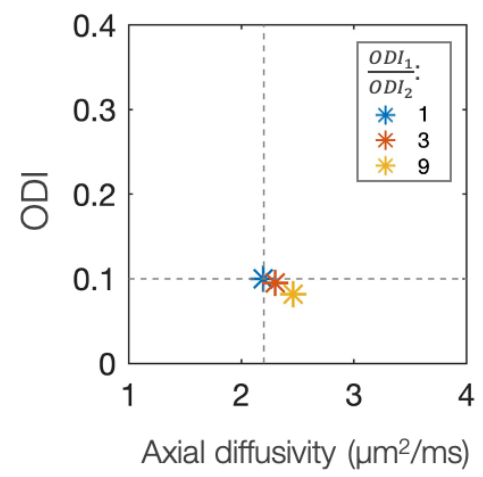

b) Intra-axonal radial diffusion



c) Extra-axonal compartment

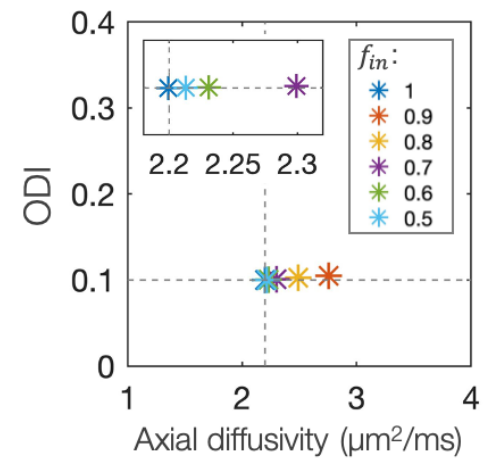

d) Two fibre populations

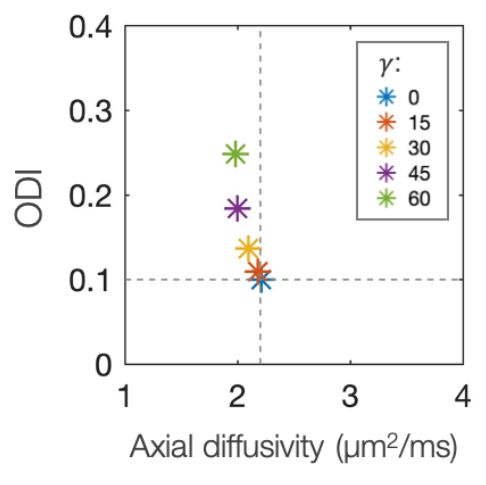

Figure 3: Violating the four assumptions of the model. Dashed lines show ground truth values. Data were simulated for a) asymmetric fibre dispersion, b) cylindrical fibres described by an axially symmetric diffusion tensor with intraaxonal radial diffusivity $d_{\perp}$, c) an extra-axonal compartment described by an axially symmetric diffusion tensor with $d_{e x, \|}=d_{i n, \|}$ and $\left.d_{e x, \perp}=d_{\|}\left(1-f_{i n}\right)[5]\right)$, and $\mathrm{d}$ ) two fibre populations of equal weighting and opening angle $\gamma$. In each case, the model was fitted assuming a Watson-like fibre distribution with symmetric dispersion, stick-like diffusion, no extra-axonal contribution and only a single fibre population per voxel. The model was fitted using the Metropolis Hastings method [26] where each point in the Figure shows the mean value of the parameter estimate distribution. Note, so that we can more easily distinguish some of the points more easily, Figure c inset shows the region $2.19<d_{\|}<2.31$ in greater detail. 
bioRxiv preprint doi: https://doi.org/10.1101/2020.10.09.332700; this version posted October 10, 2020. The copyright holder for this

preprint (which was not certified by peer review) is the author/funder, who has granted bioRxiv a license to display the preprint in perpetuity. It is made available under aCC-BY-NC-ND 4.0 International license.

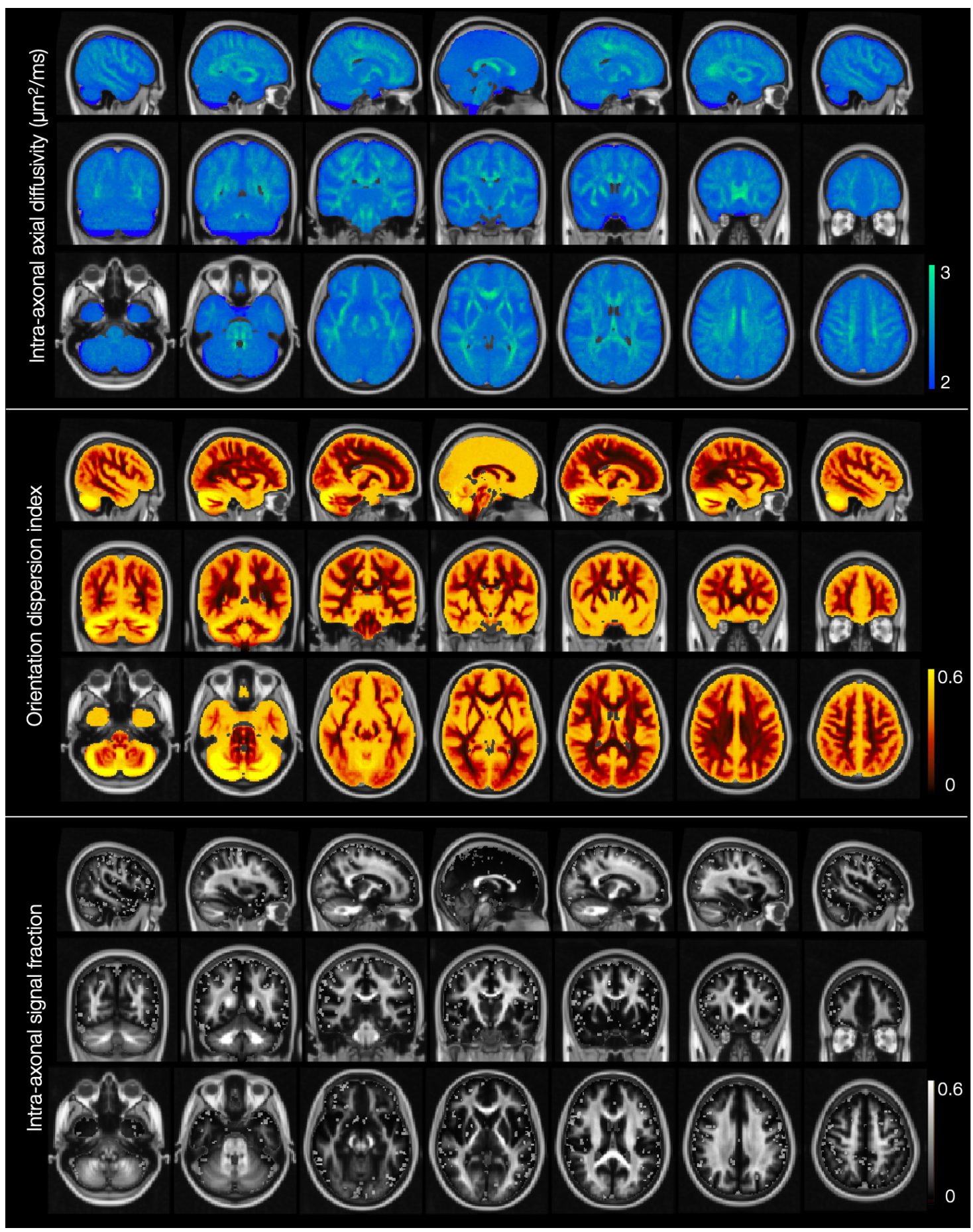

Figure 4: The intra-axonal axial diffusivity, orientation dispersion index and intra-axonal signal fraction as estimated from the dispersed stick model. The dispersed stick model was applied to data from 32 subjects and the mean parameter estimate across subjects is shown. The majority of voxels across the brain show an intra-axonal axial diffusivity of $2<d_{\|}<3 \mathrm{\mu m}^{2} / \mathrm{ms}$, where the axial diffusivity appears to vary considerably across the brain. As expected, the orientation dispersion index, ODI, is much lower in the white matter than the grey matter, with the lowest ODI values in regions of highly aligned fibres such as the corpus callosum. Assuming the T2 of the intra- and extra-axonal compartments are the same, the approximate intra-axonal signal fraction can be estimated as $F / S_{0}$. The intra-axonal signal fraction is high in the white matter and low in the grey matter. 
also to some extent in the body of the corpus callosum, the anterior external capsule, the fornix, the optic radiation and the superior cerebellar peduncle. Here the maps were clipped at $\mathrm{ODI}<0.1$ and either $d_{\|} \geq 2.6$ or $d_{\|} \geq 2.9 \mathrm{\mu m}^{2} / \mathrm{ms}$, and visually compared to the JHU ICBM-DTI-81 white matter labels [51], the JHU white matter tractography atlas [52, 53] and the Juelich histological atlas [54, 55, 56], included in FSLeyes [57] as part of the FSL suite [42].

To check whether these results were being driven by mis-registration of single subject's data to the common template, Figures 5 and 6 show the output maps from tract-based spatial statistics (TBSS) [58]. TBSS is a method for combining white matter tissue parameters across subjects, whilst avoiding artefacts due to mis-registration to the common template and partial volume effects masquerading as changes in diffusion properties. Here TBSS was used to generate a skeleton mask representing the centre of the white matter tracts. For each subject, the axial diffusivity and ODI estimated from the dispersed stick model were then projected onto the skeleton mask, after which the mean value across subjects was calculated. Note here the change in scale bar for the ODI from Figure 4. In Figure 5 we see many of the same high axial diffusivity regions as described above, giving confidence to our results. Furthermore, Figure 5 highlights regions of high axial diffusivity in the mid-body of the corpus callosum when slightly away from the midline.

In the corpus callosum (Figure 6) we see a high-low-high pattern of axial diffusivity along the anterior-posterior axis with particularly high axial diffusivity in the genu and splenium. Concurrently, we see a low-high-low pattern of dispersion along the anterior-posterior axis where a similar pattern has been previously reported [59,60]. Furthermore, we see known pattern of higher dispersion at the midline along the left-right axis of the corpus callosum [59,61], where slices 89 and 90 show elevated ODI in the midbody of the corpus callosum when compared to the more lateral slices 88 and 91.

\subsubsection{The relationship between intra-axonal axial diffusivity and ODI}

Figure 7 shows the distribution of the intra-axonal axial diffusivity and ODI for voxels in the white matter. As the dispersed stick model assumes only a single fibre population per voxel, voxels with ODI $>\sim 0.3$ should be interpreted with extreme caution and focus should be instead on the most-likely single fibre voxels with low ODI. In highly aligned fibres ODI $<\sim 0.1$ there is a negative relationship between the axial diffusivity and ODI (Figure $7 \mathrm{~b}, y=-3.14 x+2.90, r=-0.61$ ), which is less pronounced for ODI $>0.1$ i.e. the majority of voxels $(y=-0.59 x+2.64, r=-0.43)$. This behaviour was not observed in simulated data (data not shown).

\subsection{Undulations}

Figure 8 explores how fibre undulations - a form of microscopic dispersion - affect our estimated values of axial diffusivity and ODI. Here data were simulated for a simple configuration, where particles displaced along a fibre of zero radius, with undulations of a given wavelength and amplitude. Fibre undulations are seen to increase the estimated ODI and decrease the axial diffusivity from the ground truth values, where the magnitude of this effect is dependent on both the undulation amplitude and wavelength.

Our interpretation of these results is as follows. i) In the dispersed sticks model that is being fitted 
bioRxiv preprint doi: https://doi.org/10.1101/2020.10.09.332700; this version posted October 10, 2020. The copyright holder for this preprint (which was not certified by peer review) is the author/funder, who has granted bioRxiv a license to display the preprint in perpetuity. It is made available under aCC-BY-NC-ND 4.0 International license.

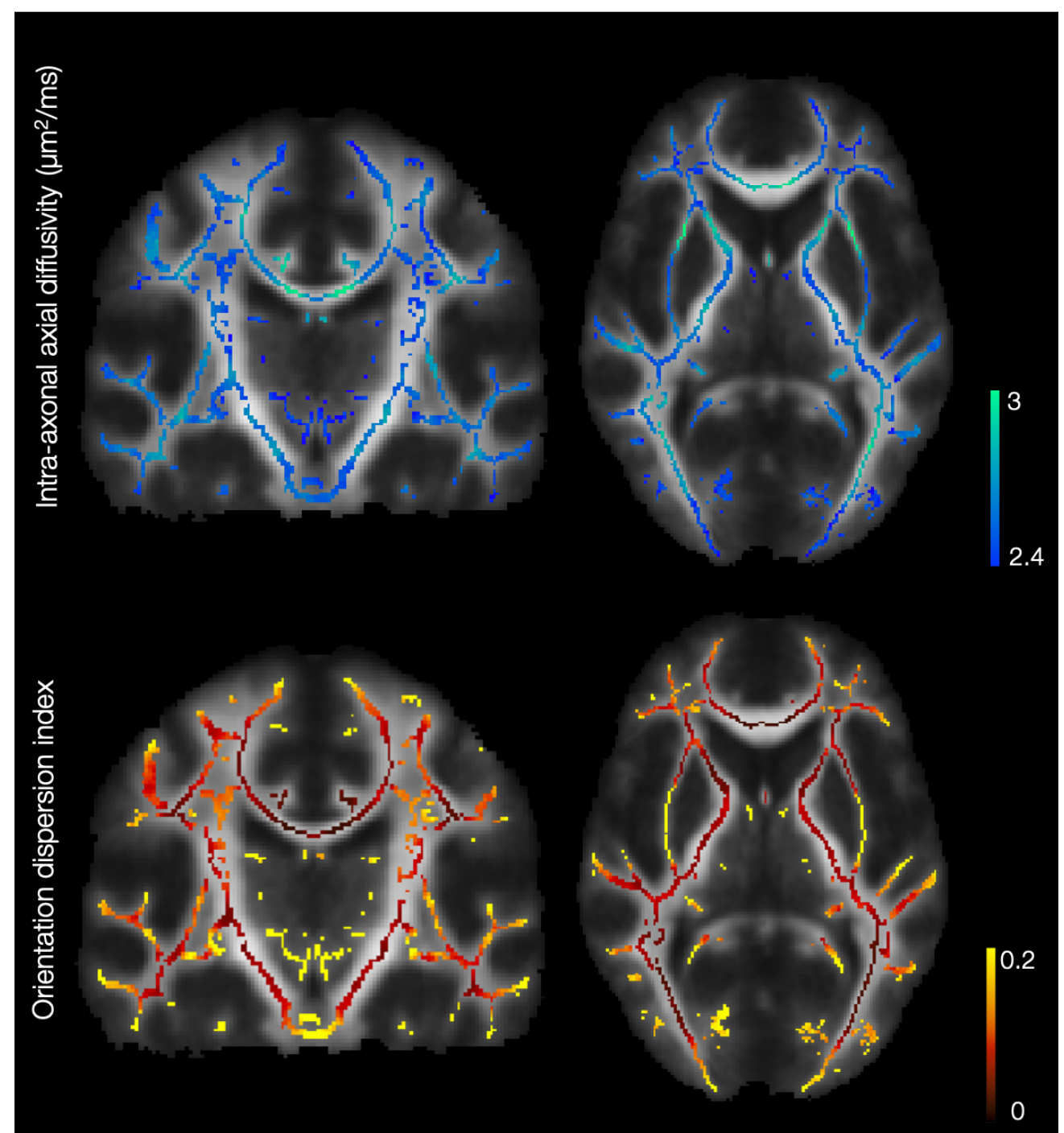

Figure 5: The intra-axonal axial diffusivity and ODI maps output from tract-based spatial statistics, TBSS [58]. Note here the change in scale bar for the ODI when compared to Figure 4. The axial diffusivity shows clear, symmetric patterns of variation across the white matter, with particularly high values in the internal capsule, external capsule, optic radiation, and in the mid-body of the corpus callosum, with slightly lower values at the midline. The ODI is particularly low in the centre of major tracts such as the corpus callosum, optic radiation and regions of the corticospinal tract. 
bioRxiv preprint doi: https://doi.org/10.1101/2020.10.09.332700; this version posted October 10, 2020. The copyright holder for this preprint (which was not certified by peer review) is the author/funder, who has granted bioRxiv a license to display the preprint in perpetuity. It is made available under aCC-BY-NC-ND 4.0 International license.
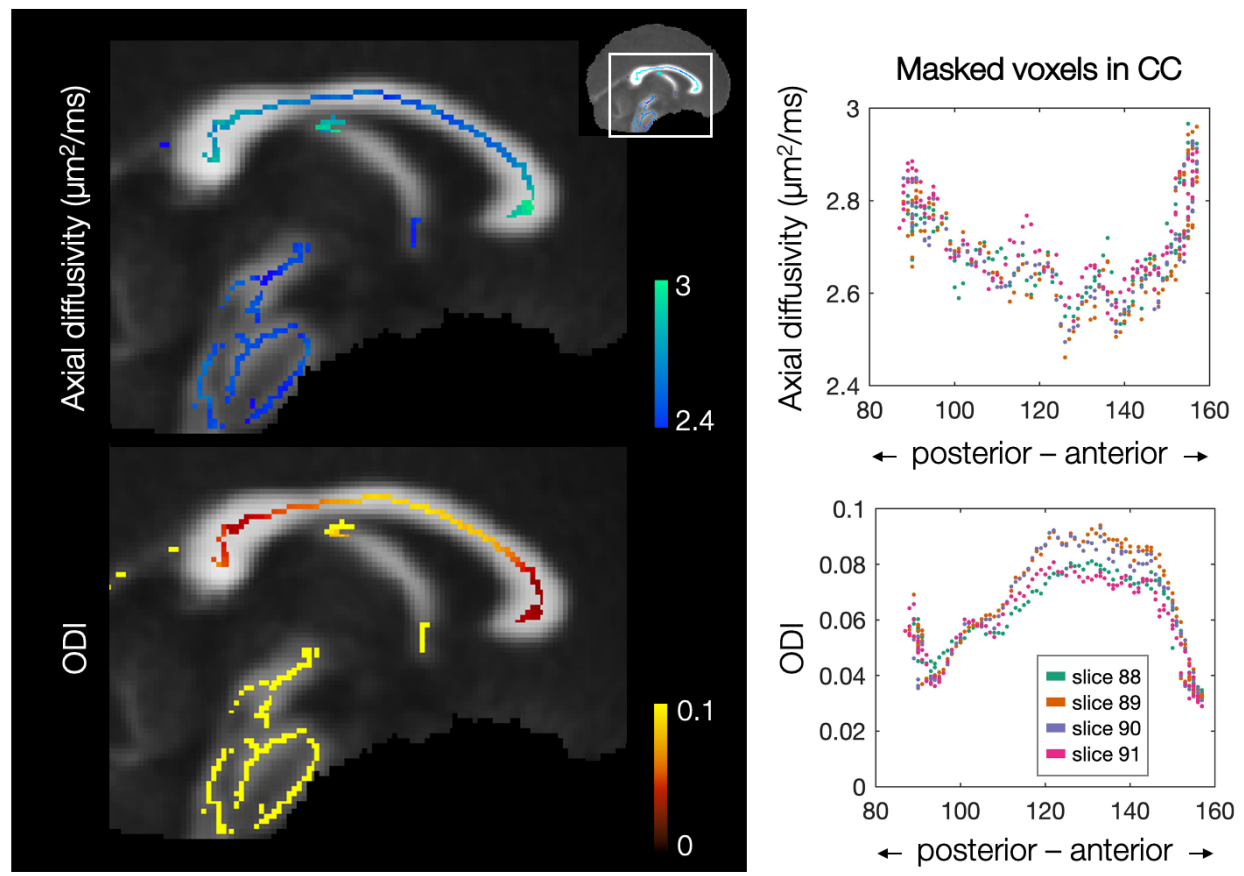

Figure 6: Variations in the intra-axonal axial diffusivity (top) and fibre orientation dispersion (bottom) across the corpus callosum. Here we show the mean parameter maps after tract-based spatial statistics, TBSS [58]. For interpretation, the mean parameter estimates (across subjects) are overlaid on a mean FA image. We see a highlow-high pattern of axial diffusivity along the anterior-posterior axis with particularly high values found in the genu (anterior) and splenium (posterior). In comparison, we see a low-high-low pattern of the orientation dispersion. Right: The axial diffusivity and ODI values plotted as a function of coordinate position along the anterior-posterior axis. The colours represent four different sagittal planes or 'slices' at the midline of the corpus callosum. Similar trends are seen across all slices where, in the midbody, we see with increased ODI at the midline (slices 89 \& 90). 
bioRxiv preprint doi: https://doi.org/10.1101/2020.10.09.332700; this version posted October 10, 2020. The copyright holder for this preprint (which was not certified by peer review) is the author/funder, who has granted bioRxiv a license to display the preprint in perpetuity. It is made available under aCC-BY-NC-ND 4.0 International license.

a)
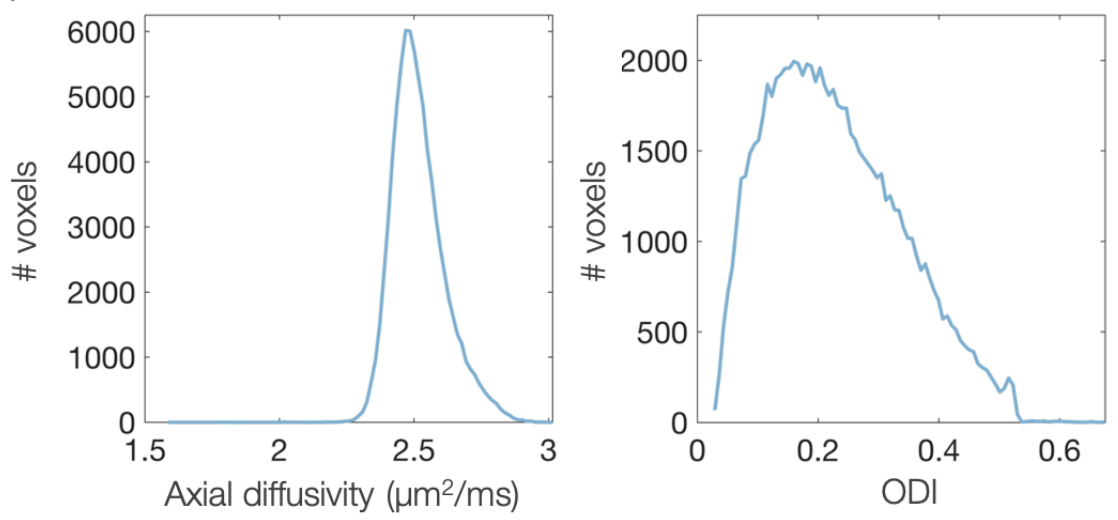

b)
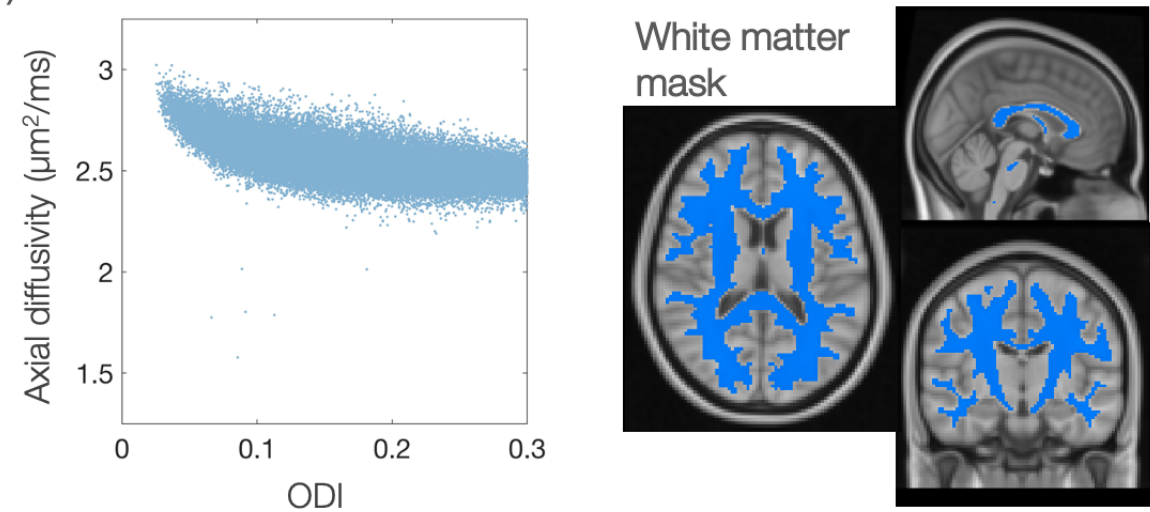

Figure 7: Variations in intra-axonal axial diffusivity and orientation dispersion index, ODI, across the white matter. a) Histograms of the mean parameter maps. b) The relationship between the estimated ODI and axial diffusivity. For $\mathrm{ODI}<\sim 0.1$ a negative correlation can be seen between the axial diffusivity and ODI, though in the majority of voxels (ODI>0.1), the parameters appear fairly uncorrelated. 
to data, the only thing that can describe diffusion orthogonal to the principal fibre orientation is dispersion. ii) Since molecules are displacing along a fibre according to the true axial diffusivity, any apparent dispersion has to be accompanied by an apparent reduction in axial diffusivity (since by definition molecules displace less along the "direction" of the fibre). iii) The amount of apparent diffusion in this direction depends on both the undulation amplitude (maximum possible displacement) and wavelength (how far they displace in practice compared to this maximum, given the true diffusivity). iv) In general, higher amplitude increases the apparent dispersion since it appears that there is more diffusion perpendicular to the principal axis. v) This apparent dispersion peaks when the characteristic diffusion length is comparable to a long stretch of an undulation, which corresponds to some "sweet spot" of amplitude and length. If the wavelength is shortened for a given amplitude, the molecules will generally "turn a corner" and displace less on average radially (i.e. less dispersion); if it is longer for that amplitude, they travel less far radially.

Finally, Figure 8 generally demonstrates a negative relationship between axial diffusivity and ODI. From Figure 8 we can conclude that the ODI parameter captures both the inter-axonal dispersion (e.g. undulations) and intra-axonal dispersion (e.g. fanning), whilst undulations hinder the displacement of water along the axon, reducing the apparent axial diffusivity. This could help explain the negative correlation between axial diffusivity and ODI in Figure 7b when ODI $<\sim 0.1$. Notably, in this simple experiment, an undulation amplitude of only $\sim 2 \mu \mathrm{m}$ can reduce the axial diffusivity from 3 to $\sim 2.5 \mathrm{\mu m}^{2} / \mathrm{ms}$, as is seen in the data (Figure $7 \mathrm{~b}$ ).

\subsection{Impact on existing models: NODDI}

To demonstrate how our results may impact the wider modelling community, Figures 9 and 10 show how the parameters of NODDI [5], one of the most commonly used microstructural models, change when the assumed axial diffusivity is changed from $d_{\|}=1.7 \mu \mathrm{m}^{2} / \mathrm{ms}$ - as is typically assumed - to $2.5 \mu \mathrm{m}^{2} / \mathrm{ms}$ (the mean axial diffusivity in the white matter) or $3 \mu \mathrm{m}^{2} / \mathrm{ms}$ (the upper limit of values estimated from the data).

Figure 9 shows an increase in the gross signal fraction associated with the intra-axonal compartment and a decrease of the extra-axonal compartment when the assumed axial diffusivity is increased from 1.7 to $3 \mu^{2} / \mathrm{ms}$. Interestingly, when $d_{\|}=3 \mu \mathrm{m}^{2} / \mathrm{ms}$, both the extra-axonal signal fraction and radial diffusivity show two distinct distributions associated with the white and grey matter. The ODI is seen to increase in both the grey and white matter, though the increase is small when $d_{\|}$is increased from $2.5 \mathrm{\mu m}^{2} / \mathrm{ms}$ to $3 \mathrm{\mu m}^{2} / \mathrm{ms}$. Finally, the signal fraction associated with isotropic diffusion, $f_{\text {iso }}$ is reduced close to zero across most of the brain and particularly in the white matter where we would not expect to find isotropic diffusion.

In Figure 10 we see the NODDI parameter maps with axial diffusivity $d_{\|}=3 \mu^{2} / \mathrm{ms}$ as a percentage of equivalent maps for $d_{\|}=1.7 \mathrm{\mu m}^{2} / \mathrm{ms}$. When $d_{\|}=3 \mu \mathrm{m}^{2} / \mathrm{ms}$, the isotropic signal fraction and extra-axonal signal fraction decrease on average to $20-40 \%$ and $\sim 50 \%$ respectively of their value when $d_{\|}=1.7 \mathrm{\mu m}^{2} / \mathrm{ms}$. Concurrently, the signal fraction associated with the intra-axonal compartment and the ODI increase on average to $\sim 150 \%$ and $\sim 120 \%$. Here we do not see a global, step change, but rather one which varies across the tissue. For example, the extra-axonal compartment is particularly reduced in the cerebellum with values at $d_{\|}=3 \mu^{2} / \mathrm{ms} 25 \%$ of their value when $d_{\|}=1.7 \mathrm{\mu m}^{2} / \mathrm{ms}\left(f_{e x}\left(d_{\|}=1.7\right) \sim 0.4, f_{e x}\left(d_{\|}=3\right) \sim 0.1\right)$. In addition, the ODI 
a)


$a m p=5 \mu m$

$l_{c}=\sqrt{2 D \Delta}=18 \mu \mathrm{m}^{2} \mid D=3 \mu \mathrm{m}^{2} / \mathrm{ms}, \Delta=55 \mathrm{~ms}$
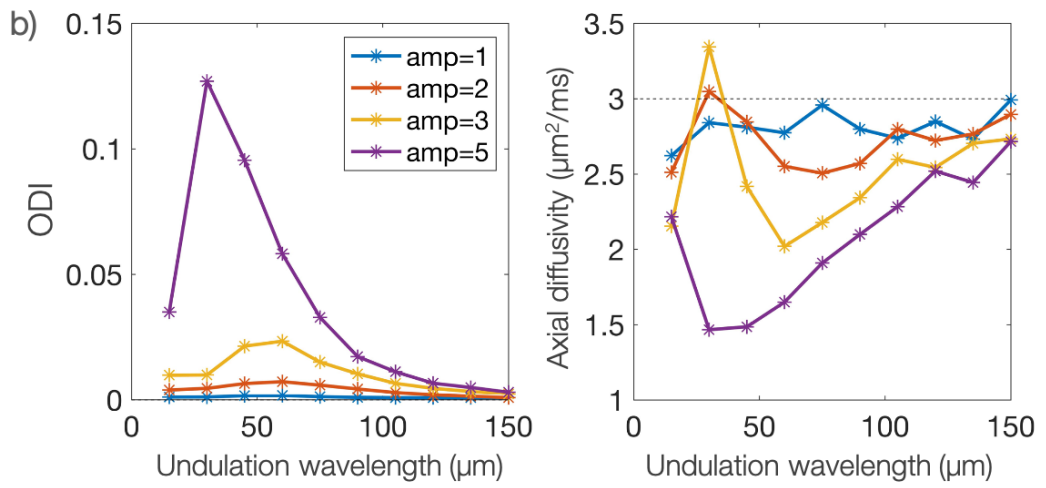

Figure 8: The effect of fibre undulations on the estimated axial diffusivity and orientation dispersion index. Here, the diffusion signal was simulated for particles confined to a fibre of zero-radius, with sinusoidal undulations of amplitude amp and wavelength $\lambda$. $D$ is the diffusion constant, $\Delta$ the diffusion time. a) Example plots of particle displacement along the fibre when $a m p=5 \mu \mathrm{m}$. b) In the presence of fibre undulations, the ODI was overestimated whilst the axial diffusivity was underestimated when compared to the ground truth values of macroscopic ODI $=0$ and $d_{\|}=3 \mu \mathrm{m}^{2} / \mathrm{ms}$ (dashed line). 

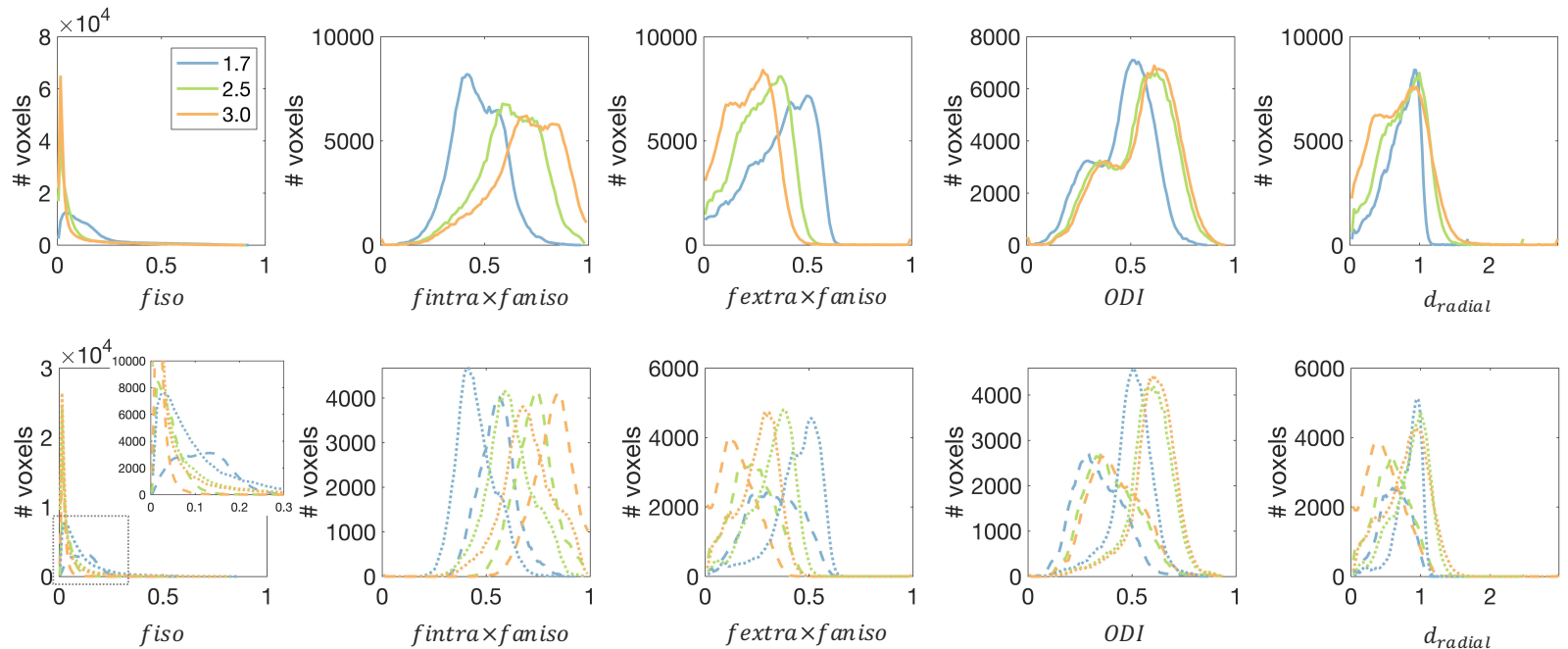

Figure 9: The dependence of NODDI parameters on the assumed axial diffusivity. The NODDI model [5] was fitted to 10 subjects from the HCP dataset with various assumed axial diffusivities $d_{\|}=1.7,2.5,3 \mu^{2} / \mathrm{ms}$. The distribution of the mean parameters is shown for all voxels in the brain (top) and for the white matter (bottom, dashed) and grey matter (bottom, dotted) separately.

is substantially increased in the corticospinal tract as well as by $\leq 250 \%$ in areas of the optic radiation and corpus callosum. Interestingly, we see a large ODI change across much of the the corpus callosum, though not at the midline along the left-right axis, a known region of increased fibre dispersion. In post mortem macaque data, substantial undulations can be seen off the midline of the corpus callosum in areas similar to the hotspots reported here [8].

\section{Discussion}

Measurement of the intra-axonal axial diffusivity is typically confounded by both signal contributions from the extra-axonal space and fibre orientation dispersion. If unaccounted for, the former will result in overestimation, and the latter underestimation, of the true axial diffusivity. For example, (from Figure 1b) at a b-value of $3 \mathrm{~ms} / \mathrm{um}^{2}$, if a fibre population with very low dispersion $(\mathrm{ODI}=0.05)$ is assumed to be perfectly aligned with zero dispersion, a ground truth axial diffusivity of $2.5 \mathrm{\mu m}^{2} / \mathrm{ms}$ is reduced to $\sim 2.2 \mathrm{\mu m}^{2} / \mathrm{ms}$. Therefore, this study utilised high b-value data to isolate the signal arising solely from the intra-axonal compartment and the "dispersed sticks model" to estimate both fibre orientation dispersion and intra-axonal axial diffusivity on a voxelwise basis.

\subsection{Comparison to previous literature}

The model was applied to high b-value in vivo data from 32 subjects where our results suggest that the axial diffusivity lies between $\sim 2.3$ and 3 in the human brain. Notably, here the upper limit is close to $\sim 3-3.1 \mathrm{\mu m}^{2} / \mathrm{ms}$, the absolute limit of water diffusion at $37^{\circ} \mathrm{C}$, though this limit was never enforced as a constraint during modelling. When compared to previous literature (see Jelescu et al $[6]$ for review, as well as $[62,16,63,12]$ ), our reported values appear fairly high. Notably, 
bioRxiv preprint doi: https://doi.org/10.1101/2020.10.09.332700; this version posted October 10, 2020. The copyright holder for this preprint (which was not certified by peer review) is the author/funder, who has granted bioRxiv a license to display the preprint in perpetuity. It is made available under aCC-BY-NC-ND 4.0 International license.

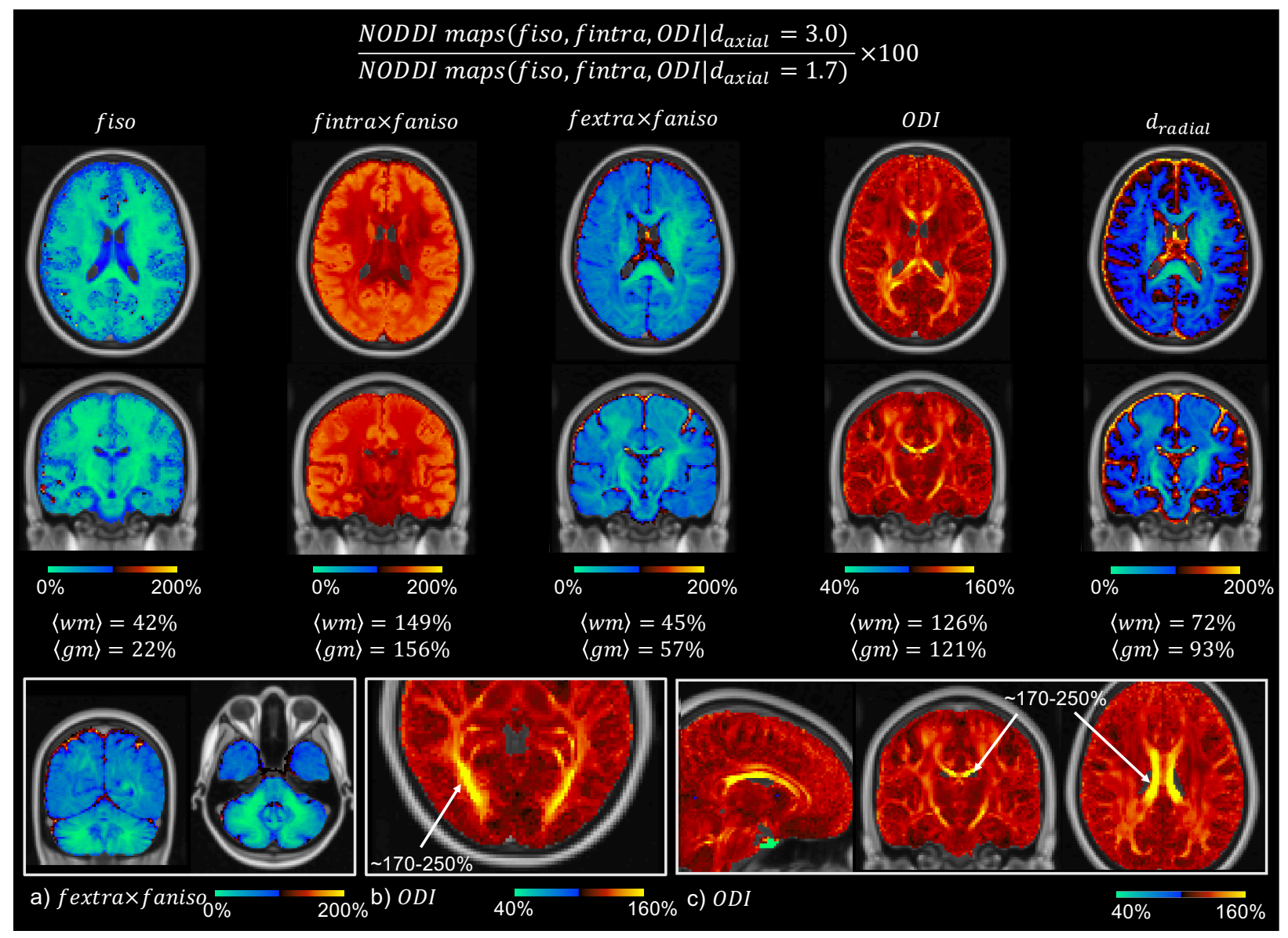

Figure 10: Estimated NODDI parameter maps with axial diffusivity $d_{\|}=3 \mu \mathrm{m}^{2} / \mathrm{ms}$ as a percentage of equivalent maps for $d_{\|}=1.7 \mathrm{~m}^{2} / \mathrm{ms}$, as is typically assumed. The signal fraction associated with isotropic diffusivity and the extra-axonal compartment is substantially reduced, as is the radial diffusivity. The intra-axonal signal fraction is largely increased (close to fintra $\sim 1$ ) as is the ODI, though to a broadly lesser extent. a) The extra-axonal compartment is reduced substantially in the cerebellum. b,c) The ODI increased to $\sim 170-250 \%$ in areas of the optic radiation and corpus callosum. 
these studies use different methods to achieve compartment specific selectivity and estimate the intra-axonal axial diffusivity to be generally in the range of $\sim 1.7-2.4 \mathrm{\mu m}^{2} / \mathrm{ms}$.

Interestingly, several previous studies have alluded to the importance of accounting for orientation dispersion when estimating axial diffusivity $[64,63]$. For example, magnetic resonance spectroscopy is often used to study the diffusion of metabolites (e.g. NAA). Assuming straight axons, Kroenke et al. [65] and Palombo et al. [66] measure the diffusivity of NAA to be $0.36 \mathrm{\mu m}^{2} / \mathrm{ms}$ in the rat and $0.33 \mathrm{\mu m}^{2} / \mathrm{ms}$ in the mouse brain respectively. In comparison Ronen et al. [64] account for the micro and macroscopic curvature of the human corpus callosum to estimate a much higher diffusivity of 0.51. As these studies consider the diffusion of NAA not water (as is reported in this study) the diffusivities should not be directly compared. More, they support our postulation that, when unaccounted for, fibre dispersion artificially lowers the estimate of axial diffusivity.

Higher diffusivities, comparable to those reported here, were previously reported by Kunz et al. [11]. Using the injection of a gadolinium(Gd)-based contrast agent to selectively reduce the signal from the extra-axonal compartment, Kunz et al. report an intra-axonal axial diffusivity close to $3 \mathrm{um}^{2} / \mathrm{ms}$ in the genu of the rat corpus callosum. In the work presented here, some of the highest values of axial diffusivity $\left(>2.9 \mathrm{\mu m}^{2} / \mathrm{ms}\right)$ were found in the genu of the corpus callosum, with the genu showing a median value of $2.76 \mathrm{\mu m}^{2} / \mathrm{ms}$ at the midline (Figure 6 ).

Our work highly complements that by Kaden et al. who observe a spatially varying axial diffusivity within a range of $\sim 2-2.7 \mathrm{~m}^{2} / \mathrm{ms}$, where the diffusivities are generally slightly lower than those reported here. We utilise high b-value data to remove the effect of extra-axonal water and directly model both the macroscopic orientation dispersion. In comparison, Kaden et al. utilise the spherical mean technique to remove the effects of macroscopic dispersion and directly model the extra-axonal space as an axially symmetric diffusion tensor, where the intra- and extra-axonal axial diffusivities are assumed equivalent and so defined by a single parameter. However, recent studies which optimise both the intra- and extra-axonal axial diffusivity independently suggest the values are likely different $[6,67]$, with intra-axonal axial diffusivity larger than extra-axonal axial diffusivity $[10,22,63]$. This would explain why we report a slightly higher diffusivities: if the apparent axial diffusivity measured by Kaden et al. represents some weighted mean from both compartments, it would likely be lower than the intra-axonal axial diffusivity estimated here. Interestingly, Kaden et al. report a lower axial diffusivity in the splenium of the corpus callosum than at the midline which contradicts the high-low-high pattern of axial diffusivity in Figure 6 .

Finally, our average diffusivity across the white matter, $\left\langle d_{\|, w m}\right\rangle=2.52 \mu \mathrm{m}^{2} / \mathrm{ms}$ is not too dissimilar to that reported by McKinnon et al. [15] who utilise high $\mathrm{b}$-value data $\left(\mathrm{b}=6 \mathrm{~ms} / \mathrm{um}^{2}\right)$ and fibre ball white matter model to estimate an intra-axonal axial diffusivity of $2.46 \mathrm{\mu m}^{2} / \mathrm{ms}$.

\subsection{Variations in axial diffusivity across the brain}

As we optimise the dispersed stick model on a voxelwise basis - rather than fitting to the average signal across many voxels - our technique shows the extent to which intra-axonal axial diffusivity varies across the brain. The axial diffusivity in the grey matter appears lower than that in the white matter (Figure 4) [68], and the intra-axonal axial diffusivity varies considerably across the white matter (Figures $4,5 \& 6$ ). In addition, we report an interesting high-low-high pattern 
of axial diffusivity across the corpus callosum which qualitatively mirrors (anti-correlates) the previously reported low-high-low pattens in axon diameter [60? , 69, 70]. This result is intriguing (see below discussion on sensitivity to axon diameter), where the relationship between the axial diffusivity and the axon diameter, alongside other aspects of the tissue microstructure, warrant further investigation.

Furthermore, the observed variations in axial diffusivity directly challenge the assumption of a global axial diffusivity as is assumed by many of the most commonly used diffusion models. Here we give two examples. Firstly, in constrained spherical deconvolution (CSD) [71], typically a global fibre response function is first estimated from the data, and then considered constant across the data to estimate the fibre orientation distribution. In previous work [8] we combined MRI and microscopy data into a joint model and showed how, when estimated on a voxelwise basis, the fibre response function appeared to vary across the white matter - results which challenge the assumption of a global fibre response function. In this previous study we were unable to separate intra- and extra-axonal contributions or estimate specific microstructural properties such as the intra-axonal axial diffusivity as we do in the work presented here. Secondly, NODDI assumes a global value of axial diffusivity, typically of $1.7 \mathrm{\mu m}^{2} / \mathrm{ms}$, to estimate the fibre orientation dispersion and 'density' i.e. the signal fraction associated with the intra-axonal compartment [5]. In both cases (CSD \& NODDI), should the assumed diffusion characteristics be wrong, all estimated parameters will be biased.

Finally, both the observed variations in intra-axonal axial diffusivity, and the sensitivity of our model to detect such fluctuations, suggest the intra-axonal axial diffusivity could act as an interesting biomarker in future studies of tissue health and disease. Indeed, Kaden et al. report significant differences in the estimated axial diffusivity in two mouse models of tuberous sclerosis [68], suggesting sensitivity of axial diffusivity to neuropathological processes. Furthermore, our simulations of fibre undulation hint to the estimated axial diffusivity being an indirect measure of fibre undulation, where the biological relevance of fibre undulations remains relatively unexplored.

\subsection{Negative correlation between axial diffusivity and orientation dispersion}

Our results show a negative correlation between axial diffusivity and orientation dispersion (Figure $7 \mathrm{~b}$ ), particularly in highly aligned fibre bundles (ODI $<\sim 0.1$ ). We first questioned whether the behaviour could be due to a degeneracy between the parameters in the model. From Figure 1b,c, we see how an underestimation of ODI results in an underestimation of axial diffusivity, suggesting a positive relationship between the variables. Furthermore, through MCMC optimisation [24], we obtain not a single parameter estimate but a parameter distribution and so could examine the covariance of any two parameters in the model. Upon examination we again found a positive correlation between axial diffusivity and ODI (data not shown). Together these results strongly suggests that model degeneracy is not the cause of the negative correlation in the data.

The negative correlation could instead be caused by a feature of the data which is unaccounted for in the model. For example, if one of the 4 modelling assumptions (c.f. Section 2.1.1) were violated. One possibility raised by our simulations is sheet-like fibre populations: Figure 3a shows how asymmetric dispersion results in a negative relationship between axial diffusivity and ODI. However, this is likely not a good explanation as it implies that low ODI voxels have highly 
asymmetric dispersion i.e. that we would have 'sheet-like' fibres across low ODI regions such as the body of the corpus callosum, the corticospinal tract and the optic radiation. Additionally, the presence of sheet-like fibres in the brain remains a controversial topic $[72,73,74]$ and their existence requires concrete validation from non-MRI methods.

Again using simulated data (Figure 3), we see how multi-fibre voxels may led to an underestimation of axial diffusivity and an over estimation of ODI (Figure 3d), where the majority of voxels in the white matter likely contain more than one fibre bundle. These results suggest the axial diffusivity should be close to that of free water, and that it appears reduced in white matter regions with multiple fibre populations per voxel. This effect could be further investigated by extending the model to account for multiple fibre populations per voxel.

It is possible that the intra-axonal axial diffusivity is overestimated due to signal contributions from the extra-axonal compartment. At high b-value, signal contributions from highly mobile water (i.e. that with considerable diffusivity) becomes negligible. However, if the extra-axonal compartment is characterised by a small radial diffusivity, this could lead to an overestimation of the intra-axonal axial diffusivity (as showed by our simulations in Figure 3c). Furthermore, our results from in vivo data show a small negative relationship between the estimated intra-axonal signal fraction $f_{i n}=F / S_{0}$ and the ODI (when ODI $<0.1, f_{\text {in }}=-1.21 \mathrm{ODI}+0.54, r=-0.27$ ) where the intraaxonal signal fraction is generally higher in low ODI voxels. $f_{i n}$ and $d_{\|}$showed no relationship (data not shown). Therefore, if low ODI voxels tend to have a higher intra-axonal signal fraction and thus contain non-negligible contributions from the extra-axonal space (where the radial diffusivity of the extra-axonal space is small), this could lead to an overestimation of the intra-axonal axial diffusivity in low ODI voxels. In this study (Figure 4) we estimate an intra-axonal signal fraction of $\sim 0.3<f_{\text {in }}<0.7$ across the deep white matter. Note, this is only a very approximate estimate as it assumes the T2 of the intra- and extra-axonal space to be the same, though it is likely different [22]. Nonetheless, if these estimates are approximately correct, assuming the extra-axonal space is well described by the tortuosity model of Figure 3c, the overestimation of the intra-axonal axially diffusivity due to extra-axonal contributions should be fairly small $(<5 \%)$.

Intuitively, the negative correlation could be due to fibre undulations [30,31, 32, 33, 34] which both act as a form of microscopic dispersion - increasing the amount of dispersion per voxel - and hinder the diffusion of intra-axonal water along the primary fibre axis, decreasing the apparent axial diffusivity. To investigate the impact of fibre undulations, we simulated data for a simple model with particles displacing along a fibre of zero radius and undulations of varying wavelength and amplitude, to find a negative relationship between the estimated orientation dispersion and axial diffusivity as expected (Figure 8). Whilst the microscopic dispersion was accounted for in the estimate of orientation dispersion, the apparent axial diffusivity was lowered with respect to the ground truth value for straight fibres. Interestingly, in this simplistic model, undulations with an amplitude of only $\sim 2 \mu \mathrm{m}$ could lower the axial diffusivity from 3 to $\sim 2.5 \mathrm{~mm}^{2} / \mathrm{ms}$, as is seen in the data (Figure $7 \mathrm{~b}$ ). In current literature, the impact of undulations often focuses on the measurement of radial diffusion or axon diameter [32, 75, 33], though the effect of undulations on other diffusion characteristics, such as axial diffusion, deserve further investigation. Future work will benefit greatly from more realistic simulations $[76,34,33]$ where, for example, the tissue structure is directly inspired by $3 \mathrm{D}$ reconstructed surfaces from microscopy images of real tissue $[77,34,33]$. 
As fibre undulations result in a lowered axial diffusivity, it follows that the observed variations in axial diffusivity may, at least to some extent, be driven by the presence of fibre undulations. Consequently, if undulations vary across the brain, there may exist a more global intra-axonal axial diffusivity. If undulations are the source of this negative correlation, then this would suggest that voxels estimated here with high axial diffusivity - i.e. regions in the corpus callosum (particularly the genu), the external capsule, the fornix, the optic radiation and the superior cerebellar peduncle - should comprise of fairly straight fibres with few undulations. Furthermore, it would imply a low-high-low pattern of undulations along the anterior-posterior axis of the corpus callosum, with large axons experiencing a higher degree of undulation. Note, this remains a highly speculative hypothesis and requires supporting evidence from, for example, maps derived from both MRI and microscopy which show the extent of fibre undulations across the brain.

\subsection{Impact: NODDI modelling}

Neurite Orientation Dispersion and Density Imaging (NODDI) [5] is one of the most commonly used microstructural models in diffusion MR which, due to its clinically feasible scan times, has featured in numerous studies of patient populations [78, 79, 80, 81, 82]. However, to estimate the neurite density or signal fraction, NODDI must assume a fixed, predefined value of the intra-axonal axial diffusivity, which is typically set to $1.7 \mathrm{\mu m}^{2} / \mathrm{ms}$. If the assumed axial diffusivity is wrong, the remaining NODDI parameters will be biased.

Our results suggest the assumed value of axial diffusivity should be between $\sim 2.5-3 \mu \mathrm{m}^{2} / \mathrm{ms}$. Figures 9 and 10 demonstrate the substantial impact this would have on the estimated NODDI maps. In Figure 10 - when $d_{\|}=3 \mu \mathrm{m}^{2} / \mathrm{ms}$ is compared to $d_{\|}=1.7 \mu \mathrm{m}^{2} / \mathrm{ms}$ - we frequently see the parameter estimates rise or fall by $\sim 50 \%$, though in extreme cases the parameter may be as little as $20 \%$ or as much as $250 \%$ of its former value. Notably, in the white matter a substantially larger signal fraction is associated with the intra-axonal compartment, whilst the signal fraction of the extra-axonal compartment is highly reduced and that associated with the isotropic compartment is reduced to $\sim 0$. Interestingly, the extra-axonal compartment is decreased from $f_{e x} \sim 0.1$ to $f_{\text {ex }} \sim 0$ across much of the corpus callosum as well as various other regions in the white matter. This suggests that the apparent signal attenuation perpendicular to the fibre can be explained solely by the interplay of intra-axonal diffusion and fibre orientation dispersion, without the need for an additional extra-axonal compartment.

We see an overall $\sim 20 \%$ increase in the orientation dispersion index, with regions of the corpus callosum and optic tract sometimes doubling, from $\sim 0.05$ to $\sim 0.1$ which invites future ODI validation against microscopy gold standards.

Importantly, the changes in the NODDI outputs reported here do not necessarily negate group differences in NODDI parameters, but rather questions the accuracy of the output values. Indeed, Schilling et al. [83] found the NODDI ODI to correlate well with dispersion values derived from histology, though without a one-to-one mapping of the values i.e. the regression line was not the line of unity. In comparison, Grussu et al. [84] compared the NODDI outputs to histological data from the human spinal chord to find an approximate one-to-one mapping. The discrepancy between the results from the two studies may indeed be related to the choice of assumed axial diffusivity - a choice that is especially challenging in post mortem tissue where the diffusivities are 
considerably reduced when compared to their in vivo values. In post mortem spinal chord, Grussu et al. assume an axial diffusivity of $1.7 \mathrm{\mu m}^{2} / \mathrm{ms}$ which was found to optimise the fitting across all samples. Schilling et al. imaged post mortem squirrel monkey brain, though the assumed axial diffusivity wasn't reported.

\subsection{Limitations}

In this study, the dispersed stick model accounts for only a single fibre population per voxel. Therefore, in our results, areas of known crossing fibres and much of the grey matter should be interpreted with caution and the focus instead placed on the 'most-likely' single-fibre voxels with a low ODI. Notably, we see some of the highest values of axial diffusivity in the low ODI voxels: the mean axial diffusivity is $2.54 \mathrm{\mu m}^{2} / \mathrm{ms}$ for voxels with $\mathrm{ODI}<0.3$, but $2.67 \mathrm{\mu m}^{2} / \mathrm{ms}$ in voxels with $\mathrm{ODI}<0.1$. In future work, the dispersed stick model could be extended to account for multiple fibre populations per voxel.

Our model is fitted to high b-value data, where much of the diffusion signal is strongly attenuated and the SNR along the fibre is very low. This results in fairly noisy maps of axial diffusivity at the single subject level. Here we report the average map across 32 subjects which reassuringly shows good smoothness and symmetry. Nonetheless, using the current model, it would be challenging to compare voxelwise changes in axial diffusivity at the level of an individual.

Although the model assumes stick-like axons with no radial diffusion, at such high b-values we may in fact be sensitive to radial diffusion from the extra-axonal space (as described above) or from large diameter axons. From in vivo human data, Veraart et al. [21] estimated a radial diffusivity of $\sim 0.01 \mathrm{\mu m}^{2} / \mathrm{ms}$ across the white matter. Here, using simulated data we found that tensor-like (rather than stick-like) fibres with a radial diffusivity of $\sim 0.01 \mathrm{um}^{2} / \mathrm{ms}$ led to the over estimation of axial diffusivity, from $d_{\|}=2.2 \mathrm{\mu m}^{2} / \mathrm{ms}$ to $d_{\|}=2.68 \mathrm{\mu m}^{2} / \mathrm{ms}$ (Figure $3 \mathrm{~b}$ ). Notably, the overestimation of the axial diffusivity increased with increasing radial diffusivity (i.e. increasing axon diameter). Sensitivity to axon diameter could therefore likely explain why our estimates of axial diffusivity are higher than many previous studies. However, this possibility would not be consistent with the high-low-high pattern of axial diffusivity across the corpus callosum. The corpus callosum is known to exhibit a low-high-low pattern of axon diameter [60? , 69, 70], from which we would expect a low-high-low pattern of axial diffusivity. The opposite is reported in the data. Nonetheless, the role of axon diameter on the estimated axial diffusivity in high b-value data warrant further investigation.

Finally, the model does not account for a slowly diffusing isotropic compartment, often referred to as the 'dot compartment', the existence of which would bias our results. Tax et al. [85] recently found evidence for a dot compartment in the cerebellar grey matter accounting for $9.7 \%$ of the signal with a diffusivity of $d_{\text {iso }}=0.12 \mu \mathrm{m}^{2} / \mathrm{ms}$. A smaller contribution was also observed in the white matter of the cerebellum $\left(5.4 \%, d_{i s o}=0.16 \mathrm{~m}^{2} / \mathrm{ms}\right)$ and for some voxels in the occipital lobe $\left(2.6 \%, d_{i s o}=0.23 \mathrm{\mu m}^{2} / \mathrm{ms}\right)$. Should a dot compartment exist, the axial diffusivity would be over-estimated. In simulated data with ground truth $d_{\|}=2 \mu \mathrm{m}^{2} / \mathrm{ms}$, a dot compartment with $d_{\text {iso }}=0.2 \mathrm{\mu m}^{2} / \mathrm{ms}$ led to an estimated axial diffusivity of $\sim 2.3 \mu \mathrm{m}^{2} / \mathrm{ms}$ when the dot signal fraction was $f_{d o t}=0.05$ (as in the cerebellar white matter) and $\sim 2.1 \mu \mathrm{m}^{2} / \mathrm{ms}$ when $f_{d o t}=0.025$ (occipital white matter) i.e. a dot compartment leads to an overestimation of axial diffusivity, where the 
degree of overestimation is dependent on the volume fraction. Therefore, in our results the axial diffusivities in the cerebellum and regions of the occipital lobe may be somewhat inflated from their true value. As we do not see a clear bias with large axial diffusivities in these regions - if anything the model estimates a lower-than-typical axial diffusivities in these regions - the effect may be small.

\subsection{Code availability and future work}

The dispersed stick model was implemented both in MATLAB and the cuDIMOT framework [24] for GPU acceleration, both of which are available at [link to be added upon publication]. We provide a cuDIMOT implementation of NODDI where the assumed diffusivities $d_{\|}$and $d_{i s o}$ are user defined at runtime. Interesting avenues for future work include: the relationship between axial diffusivity and features of the tissue architecture such as axon diameter; how intra-axonal axial diffusivity is affected by tissue degradation or disease; the relationship between axial diffusivity and brain development; as well as the estimation of axial diffusivity in post mortem tissue, both in situ and after perfusion or immersion fixation. Furthermore, the model could be extended to estimate multiple fibre populations per voxel, thus improving the interpretability of the model in multi-fibre voxels. Finally, co-registered MRI and microscopy data should be used to validate the orientation dispersion estimates reported here. This will likely require the acquisition of a bespoke post mortem dataset which combines data from 3 shells at ultra-high b-values (accounting for the reduced diffusivity of fixed post mortem tissue) with corresponding microscopy imaging of the white matter fibres.

\section{Conclusion}

Through the simultaneous analysis of orientation dispersion and axial diffusivity we demonstrate substantial variability in the intra-axonal axial diffusivity across the brain. The estimated axial diffusivity ranges from $\sim 2.3-3 \mu^{2} / \mathrm{ms}$ with a mean value of $2.52 \mu \mathrm{m}^{2} / \mathrm{ms}$ in the white matter. This is considerably higher than most of the reported values in current literature, with the upper limit close to the absolute maximum diffusivity of pure water at $37^{\circ} \mathrm{C}, \sim 3-3.1 \mu \mathrm{m}^{2} / \mathrm{ms}$. Our results show how axial diffusivity varies considerably both across the corpus callosum - with particularly high values found in the genu and splenium - and between white matter tracts. This not only questions the assumption of a global axial diffusivity $[5,7]$ but also suggests that axial diffusivity could act as an interesting biomarker of disease. Furthermore, we find a negative correlation between axial diffusivity and the orientation dispersion, and suggest this could be explained by the presence of fibre undulations which act to lower the apparent axial diffusivity. Finally, we show how the estimated parameters of the NODDI model [5] changed substantially, when the assumed axial diffusivity was increased from 1.7 to 2.5 or $3 \mu \mathrm{m}^{2} / \mathrm{ms}$, as our results suggest.

\section{Acknowledgements}

Many thanks to Jelle Veraart for insightful discussions on how axon radius may affect the results presented. The data were acquired at the UK National Facility for In Vivo MR Imaging of Human 

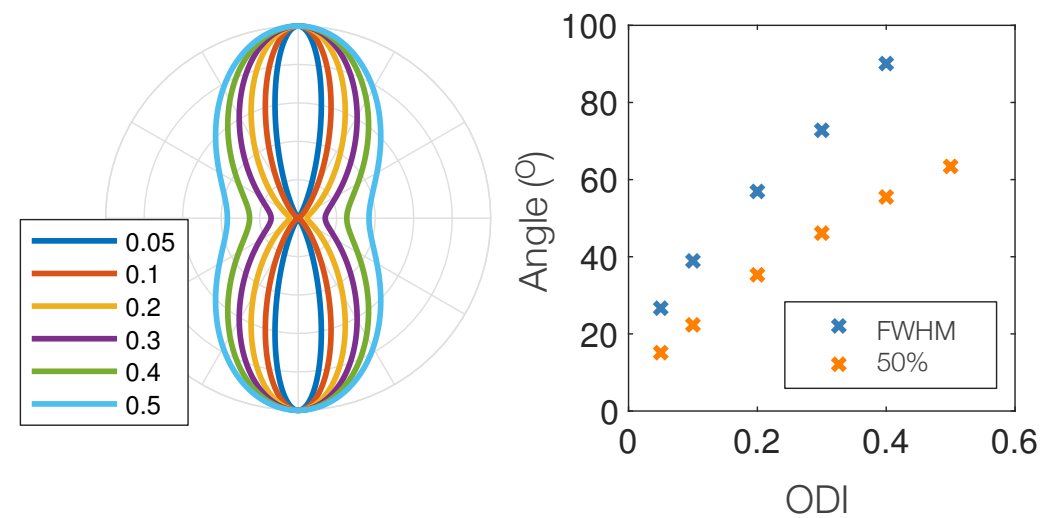

Figure 11: How the orientation dispersion index relates to the fibre opening angle in degrees. Left: A 2D plane through the fibre orientation distribution as the ODI is varied from 0.05-0.5. Right: The ODI is plotted against the full width half maximum of the fibre orientation distribution, as well as the angle containing $50 \%$ of the fibres.

Tissue Microstructure funded by the EPSRC (grant EP/M029778/1), and The Wolfson Foundation. AFDH is supported by the EPSRC and MRC (grants EP/L016052/1 and MR/L009013/1). FJL is supported by the Oppenheimer Memorial Trust and St Catherines College Oxford. DKJ is supported by a Wellcome Trust Investigator Award $(096646 / \mathrm{Z} / 11 / \mathrm{Z})$ and DKJ and MD by a Wellcome Trust Strategic Award (104943/Z/14/Z). KLM and SJ are supported by the Wellcome Trust (grants WT202788/Z/16/A and WT215573/Z/19/Z). The Wellcome Centre for Integrative Neuroimaging is supported by core funding from the Wellcome Trust $(203139 / Z / 16 / Z)$.

\section{Appendices}

\subsection{Appendix A: converting ODI to degrees}

The orientation dispersion can be described in terms of orientation dispersion index (ODI) or degrees, where there does not appear to be consistency throughout the literature. To aid interpretation of our results (quoted in ODI) and facilitate comparison to other literature, Figure 11 relates the ODI to the amount of dispersion in degrees: the full width half maximum (FWHM) of the distribution and the angle which contains $50 \%$ of all fibres.

[1] S. Jbabdi, S. N. Sotiropoulos, S. N. Haber, D. C. Van Essen, T. E. Behrens, Measuring macroscopic brain connections in vivo, Nature Neuroscience 18 (11) (2015) 1546-1555. doi:10.1038/nn.4134.

[2] D. C. Alexander, T. B. Dyrby, M. Nilsson, H. Zhang, Imaging brain microstructure with diffusion MRI: practicality and applications, NMR in Biomedicine 32 (4). doi:10.1002/nbm.3841.

[3] I. O. Jelescu, M. D. Budde, Design and Validation of Diffusion MRI Models of White Matter, Frontiers in Physics 5 (2017) 61. doi:10.3389/fphy.2017.00061.

[4] D. S. Novikov, E. Fieremans, S. N. Jespersen, V. G. Kiselev, Quantifying brain microstructure with diffusion MRI: Theory and parameter estimation, NMR in Biomedicine 32 (4) (2018) e3998. doi:10.1002/nbm.3998.

[5] H. Zhang, T. Schneider, C. A. Wheeler-Kingshott, D. C. Alexander, NODDI: Practical in vivo neurite orientation dispersion and density imaging of the human brain, NeuroImage 61 (4) (2012) 1000-1016. doi:10.1016/j.neuroimage.2012.03.072. 
[6] I. O. Jelescu, J. Veraart, E. Fieremans, D. S. Novikov, Degeneracy in model parameter estimation for multicompartmental diffusion in neuronal tissue, NMR in Biomedicine 29 (1) (2016) 33-47. doi:10.1002/nbm.3450.

[7] J. D. Tournier, C. H. Yeh, F. Calamante, K. H. Cho, A. Connelly, C. P. Lin, Resolving crossing fibres using constrained spherical deconvolution: Validation using diffusion-weighted imaging phantom data, NeuroImage 42 (2) (2008) 617-625. doi:10.1016/j.neuroimage.2008.05.002.

[8] A. Howard, S. Jbabdi, K. AA, J. Sallet, G. Daubney, J. Mollink, C. Scott, N. Sibson, K. Miller, The BigMac dataset: ultra-high angular resolution diffusion imaging and multi-contrast microscopy of a whole macaque brain, ISMRM 27th Annual Meeting.

[9] K. Schilling, Y. Gao, V. Janve, I. Stepniewska, B. A. Landman, A. W. Anderson, Confirmation of a gyral bias in diffusion MRI fiber tractography, Human Brain Mapping 39 (3) (2018) 1449-1466. doi:10.1002/hbm.23936.

[10] S. N. Jespersen, J. L. Olesen, B. Hansen, N. Shemesh, Diffusion time dependence of microstructural parameters in fixed spinal cord, NeuroImage 182 (2018) 329-342. doi:10.1016/j.neuroimage.2017.08.039.

[11] N. Kunz, A. R. da Silva, I. O. Jelescu, Intra- and extra-axonal axial diffusivities in the white matter: Which one is faster?, NeuroImage 181 (2018) 314-322. doi:10.1016/j.neuroimage.2018.07.020.

[12] B. Lampinen, F. Szczepankiewicz, J. Mårtensson, D. Westen, O. Hansson, C. Westin, M. Nilsson, Towards unconstrained compartment modeling in white matter using diffusionrelaxation MRI with tensorvalued diffusion encoding, Magnetic Resonance in Medicine 84 (3) (2020) 1605-1623. doi:10.1002/mrm.28216.

[13] J. H. Jensen, G. Russell Glenn, J. A. Helpern, Fiber ball imaging, NeuroImage 127 (A) (2016) $824-833$. doi:10.1016/j.neuroimage.2015.09.049.

[14] E. T. McKinnon, J. H. Jensen, G. R. Glenn, J. A. Helpern, Dependence on b-value of the directionaveraged diffusion-weighted imaging signal in brain, Magnetic Resonance Imaging 36 (2017) 121-127. doi:10.1016/j.mri.2016.10.026.

[15] E. T. McKinnon, J. A. Helpern, J. H. Jensen, Modeling white matter microstructure with fiber ball imaging, NeuroImage 176 (2018) 11-21. doi:10.1016/j.neuroimage.2018.04.025.

[16] J. Veraart, E. Fieremans, D. S. Novikov, On the scaling behavior of water diffusion in human brain white matter, NeuroImage 185 (2019) 379-387. doi:10.1016/j.neuroimage.2018.09.075.

[17] E. T. McKinnon, J. H. Jensen, Measuring intra-axonal T 2 in white matter with direction-averaged diffusion MRI, Magnetic Resonance in Medicine 81 (5) (2019) 2985-2994. doi:10.1002/mrm.27617.

[18] E. Kleban, C. M. Tax, U. S. Rudrapatna, D. K. Jones, R. Bowtell, Strong diffusion gradients allow the separation of intra- and extra-axonal gradient-echo signals in the human brain, NeuroImage 217 (2020) 116793. doi:10.1016/j.neuroimage.2020.116793.

[19] M. Frigo, R. H. Fick, M. Zucchelli, S. Deslauriers-Gauthier, R. Deriche, Multi Tissue Modelling of Diffusion MRI Signal Reveals Volume Fraction Bias, in: Proceedings - International Symposium on Biomedical Imaging, 2020. doi:10.1109/ISBI45749.2020.9098649

[20] K. V. Mardia, P. E. Jupp, Directional statistics, J. Wiley, 2000.

[21] J. Veraart, D. Nunes, U. Rudrapatna, E. Fieremans, D. K. Jones, D. S. Novikov, N. Shemesh, Noninvasive quantification of axon radii using diffusion MRI, eLife 9. doi:10.7554/eLife.49855.

[22] J. Veraart, D. S. Novikov, E. Fieremans, TE dependent Diffusion Imaging (TEdDI) distinguishes between compartmental T2 relaxation times, NeuroImage 182 (2018) 360-369. doi:10.1016/j.neuroimage.2017.09.030.

[23] M. Nilsson, J. Lätt, D. Van Westen, S. Brockstedt, S. Lasič, F. Ståhlberg, D. Topgaard, Noninvasive mapping of water diffusional exchange in the human brain using filter-exchange imaging, Magnetic Resonance in Medicine 69 (6) (2013) 1572-1580. doi:10.1002/mrm.24395.

[24] M. Hernandez-Fernandez, I. Reguly, S. Jbabdi, M. Giles, S. Smith, S. N. Sotiropoulos, Using GPUs to accelerate computational diffusion MRI: From microstructure estimation to tractography and connectomes, NeuroImage 188 (2019) 598-615. doi:10.1016/j.neuroimage.2018.12.015.

[25] D. K. Jones, P. J. Basser, "Squashing peanuts and smashing pumpkins": How noise distorts diffusion-weighted MR data, Magnetic Resonance in Medicine 52 (5) (2004) 979-993. doi:10.1002/mrm.20283.

[26] W. K. Hastings, Monte Carlo sampling methods using Markov chains and their applications, Biometrika 57 (1) (1970) 97-109. doi:10.1093/biomet/57.1.97.

[27] C. Bingham, An Antipodally Symmetric Distribution on the Sphere, Tech. Rep. 6 (1974).

[28] S. N. Sotiropoulos, T. E. Behrens, S. Jbabdi, Ball and rackets: Inferring fiber fanning from diffusion-weighted MRI, NeuroImage 60 (2) (2012) 1412-1425. doi:10.1016/j.neuroimage.2012.01.056.

[29] M. Tariq, T. Schneider, D. C. Alexander, C. A. Gandini Wheeler-Kingshott, H. Zhang, Bingham-NODDI: Mapping anisotropic orientation dispersion of neurites using diffusion MRI, NeuroImage 133 (2016) 207-223. doi:10.1016/j.neuroimage.2016.01.046.

[30] M. Nilsson, J. Lätt, F. Ståhlberg, D. Westen, H. Hagslätt, The importance of axonal undulation in dif- 
fusion MR measurements: a Monte Carlo simulation study, NMR in Biomedicine 25 (5) (2012) 795-805. doi:10.1002/nbm.1795.

[31] E. Özarslan, C. Yolcu, M. Herberthson, H. Knutsson, C. F. Westin, Influence of the size and curvedness of neural projections on the orientationally averaged diffusion MR signal, Frontiers in Physics 6 (2018) 17. doi:10.3389/fphy.2018.00017.

[32] J. Brabec, S. Lasič, M. Nilsson, Timedependent diffusion in undulating thin fibers: Impact on axon diameter estimation, NMR in Biomedicine 33 (3). doi:10.1002/nbm.4187.

[33] H.-H. Lee, S. N. Jespersen, E. Fieremans, D. S. Novikov, The impact of realistic axonal shape on axon diameter estimation using diffusion MRI, NeuroImage 223 (2020) 117228. doi:10.1016/j.neuroimage.2020.117228.

[34] H. H. Lee, A. Papaioannou, S. L. Kim, D. S. Novikov, E. Fieremans, A time-dependent diffusion MRI signature of axon caliber variations and beading, Communications Biology 3 (1) (2020) 1-13. doi:10.1038/s42003-0201050-x.

[35] J. A. McNab, B. L. Edlow, T. Witzel, S. Y. Huang, H. Bhat, K. Heberlein, T. Feiweier, K. Liu, B. Keil, J. CohenAdad, M. D. Tisdall, R. D. Folkerth, H. C. Kinney, L. L. Wald, The Human Connectome Project and beyond: Initial applications of 300mT/m gradients, NeuroImage 80 (2013) 234-245. doi:10.1016/j.neuroimage.2013.05.074.

[36] D. K. Jones, D. C. Alexander, R. Bowtell, M. Cercignani, F. Dell'Acqua, D. J. McHugh, K. L. Miller, M. Palombo, G. J. Parker, U. S. Rudrapatna, C. M. Tax, Microstructural imaging of the human brain with a super-scanner': 10 key advantages of ultra-strong gradients for diffusion MRI, NeuroImage 182 (2018) 8-38. doi:10.1016/j.neuroimage.2018.05.047.

[37] Y. Zhang, M. Brady, S. Smith, Segmentation of brain MR images through a hidden Markov random field model and the expectation-maximization algorithm, IEEE Transactions on Medical Imaging 20 (1) (2001) 45-57. doi:10.1109/42.906424.

[38] J. L. R. Andersson, M. Jenkinson, S. Smith, Non-linear registration aka Spatial normalisation FMRIB Technial Report TR07JA2, Tech. rep. (2007).

[39] M. W. Woolrich, S. Jbabdi, B. Patenaude, M. Chappell, S. Makni, T. Behrens, C. Beckmann, M. Jenkinson, S. M. Smith, Bayesian analysis of neuroimaging data in FSL., NeuroImage 45 (1 Suppl) (2009) S173-S186. doi:10.1016/j.neuroimage.2008.10.055.

[40] K. Setsompop, B. A. Gagoski, J. R. Polimeni, T. Witzel, V. J. Wedeen, L. L. Wald, Blipped-controlled aliasing in parallel imaging for simultaneous multislice echo planar imaging with reduced g-factor penalty, Magnetic Resonance in Medicine 67 (5) (2012) 1210-1224. doi:10.1002/mrm.23097.

[41] J. L. Andersson, S. Skare, J. Ashburner, How to correct susceptibility distortions in spin-echo echo-planar images: Application to diffusion tensor imaging, NeuroImage 20 (2) (2003) 870-888. doi:10.1016/S1053-8119(03)00336-7.

[42] S. M. Smith, M. Jenkinson, M. W. Woolrich, C. F. Beckmann, T. E. Behrens, H. Johansen-Berg, P. R. Bannister, M. De Luca, I. Drobnjak, D. E. Flitney, R. K. Niazy, J. Saunders, J. Vickers, Y. Zhang, N. De Stefano, J. M. Brady, P. M. Matthews, Advances in functional and structural MR image analysis and implementation as FSL, in: NeuroImage, Vol. 23, Neuroimage, 2004. doi:10.1016/j.neuroimage.2004.07.051.

[43] J. L. Andersson, M. S. Graham, E. Zsoldos, S. N. Sotiropoulos, Incorporating outlier detection and replacement into a non-parametric framework for movement and distortion correction of diffusion MR images, NeuroImage 141 (2016) 556-572. doi:10.1016/j.neuroimage.2016.06.058.

[44] J. L. Andersson, S. N. Sotiropoulos, An integrated approach to correction for off-resonance effects and subject movement in diffusion MR imaging, NeuroImage 125 (2016) 1063-1078. doi:10.1016/j.neuroimage.2015.10.019.

[45] D. C. Van Essen, S. M. Smith, D. M. Barch, T. E. Behrens, E. Yacoub, K. Ugurbil, The WU-Minn Human Connectome Project: An overview, NeuroImage 80 (2013) 62-79. doi:10.1016/j.neuroimage.2013.05.041.

[46] M. F. Glasser, S. N. Sotiropoulos, J. A. Wilson, T. S. Coalson, B. Fischl, J. L. Andersson, J. Xu, S. Jbabdi, M. Webster, J. R. Polimeni, D. C. Van Essen, M. Jenkinson, The minimal preprocessing pipelines for the Human Connectome Project, NeuroImage 80 (2013) 105-124. doi:10.1016/j.neuroimage.2013.04.127.

[47] S. N. Sotiropoulos, S. Jbabdi, J. Xu, J. L. Andersson, S. Moeller, E. J. Auerbach, M. F. Glasser, M. Hernandez, G. Sapiro, M. Jenkinson, D. A. Feinberg, E. Yacoub, C. Lenglet, D. C. Van Essen, K. Ugurbil, T. E. Behrens, Advances in diffusion MRI acquisition and processing in the Human Connectome Project, NeuroImage 80 (2013) 125-143. doi:10.1016/j.neuroimage.2013.05.057.

[48] M. Jenkinson, S. Smith, A global optimisation method for robust affine registration of brain images, Medical Image Analysis 5 (2) (2001) 143-156. doi:10.1016/S1361-8415(01)00036-6.

[49] M. Jenkinson, P. Bannister, M. Brady, S. Smith, Improved optimization for the robust and accurate linear registration and motion correction of brain images, NeuroImage 17 (2) (2002) 825-841. doi:10.1016/S10538119(02)91132-8.

[50] B. Lampinen, F. Szczepankiewicz, M. Novén, D. van Westen, O. Hansson, E. Englund, J. Mårtensson, C. F. 
Westin, M. Nilsson, Searching for the neurite density with diffusion MRI: Challenges for biophysical modeling, Human Brain Mapping 40 (8) (2019) 2529-2545. doi:10.1002/hbm.24542.

[51] S. S. Mori, B. J. Crain, MRI atlas of human white matter, Elsevier, 2005.

[52] S. Wakana, A. Caprihan, M. M. Panzenboeck, J. H. Fallon, M. Perry, R. L. Gollub, K. Hua, J. Zhang, H. Jiang, P. Dubey, A. Blitz, P. van Zijl, S. Mori, Reproducibility of quantitative tractography methods applied to cerebral white matter, NeuroImage 36 (3) (2007) 630-644. doi:10.1016/j.neuroimage.2007.02.049.

[53] K. Hua, J. Zhang, S. Wakana, H. Jiang, X. Li, D. S. Reich, P. A. Calabresi, J. J. Pekar, P. C. van Zijl, S. Mori, Tract probability maps in stereotaxic spaces: Analyses of white matter anatomy and tract-specific quantification, NeuroImage 39 (1) (2008) 336-347. doi:10.1016/j.neuroimage.2007.07.053.

[54] S. B. Eickhoff, K. E. Stephan, H. Mohlberg, C. Grefkes, G. R. Fink, K. Amunts, K. Zilles, A new SPM toolbox for combining probabilistic cytoarchitectonic maps and functional imaging data, NeuroImage 25 (4) (2005) 1325-1335. doi:10.1016/j.neuroimage.2004.12.034.

[55] S. B. Eickhoff, S. Heim, K. Zilles, K. Amunts, Testing anatomically specified hypotheses in functional imaging using cytoarchitectonic maps, NeuroImage 32 (2) (2006) 570-582. doi:10.1016/j.neuroimage.2006.04.204.

[56] S. B. Eickhoff, T. Paus, S. Caspers, M. H. Grosbras, A. C. Evans, K. Zilles, K. Amunts, Assignment of functional activations to probabilistic cytoarchitectonic areas revisited, NeuroImage 36 (3) (2007) 511-521. doi:10.1016/j.neuroimage.2007.03.060.

[57] P. McCarthy, FSLeyes, Zenododoi:10.5281/ZENODO.3858136.

[58] S. M. Smith, M. Jenkinson, H. Johansen-Berg, D. Rueckert, T. E. Nichols, C. E. Mackay, K. E. Watkins, O. Ciccarelli, M. Z. Cader, P. M. Matthews, T. E. Behrens, Tract-based spatial statistics: Voxelwise analysis of multi-subject diffusion data, NeuroImage 31 (4) (2006) 1487-1505. doi:10.1016/j.neuroimage.2006.02.024.

[59] M. D. Budde, J. Annese, Quantification of anisotropy and fiber orientation in human brain histological sections, Frontiers in Integrative Neuroscience 7 (2013) 3. doi:10.3389/fnint.2013.00003.

[60] H. Zhang, P. L. Hubbard, G. J. Parker, D. C. Alexander, Axon diameter mapping in the presence of orientation dispersion with diffusion MRI, NeuroImage 56 (3) (2011) 1301-1315. doi:10.1016/j.neuroimage.2011.01.084.

[61] J. Mollink, M. Kleinnijenhuis, A. M. van Cappellen van Walsum, S. N. Sotiropoulos, M. Cottaar, C. Mirfin, M. P. Heinrich, M. Jenkinson, M. Pallebage-Gamarallage, O. Ansorge, S. Jbabdi, K. L. Miller, Evaluating fibre orientation dispersion in white matter: Comparison of diffusion MRI, histology and polarized light imaging, NeuroImage 157 (2017) 561-574. doi:10.1016/j.neuroimage.2017.06.001.

[62] K. Zheng, T. P. Jensen, L. P. Savtchenko, J. A. Levitt, K. Suhling, D. A. Rusakov, Nanoscale diffusion in the synaptic cleft and beyond measured with time-resolved fluorescence anisotropy imaging, Scientific Reports 7 (1) (2017) 1-10. doi:10.1038/srep42022.

[63] B. Dhital, M. Reisert, E. Kellner, V. G. Kiselev, Intra-axonal diffusivity in brain white matter, NeuroImage 189 (2019) 543-550. doi:10.1016/j.neuroimage.2019.01.015.

[64] I. Ronen, M. Budde, E. Ercan, J. Annese, A. Techawiboonwong, A. Webb, Microstructural organization of axons in the human corpus callosum quantified by diffusion-weighted magnetic resonance spectroscopy of N-acetylaspartate and post-mortem histology, Brain structure \& function 219 (5) (2014) 1773-1785. doi:10.1007/s00429-013-0600-0.

[65] C. D. Kroenke, J. J. Ackerman, D. A. Yablonskiy, On the nature of the NAA diffusion attenuated MR signal in the central nervous system, Magnetic Resonance in Medicine 52 (5) (2004) 1052-1059. doi:10.1002/mrm.20260.

[66] M. Palombo, C. Ligneul, J. Valette, Modeling diffusion of intracellular metabolites in the mouse brain up to very high diffusionweighting: Diffusion in long fibers (almost) accounts for nonmonoexponential attenuation, Magnetic Resonance in Medicine 77 (1) (2017) 343-350. doi:10.1002/mrm.26548.

[67] D. S. Novikov, J. Veraart, I. O. Jelescu, E. Fieremans, Rotationally-invariant mapping of scalar and orientational metrics of neuronal microstructure with diffusion MRI, NeuroImage 174 (2018) 518-538. doi:10.1016/j.neuroimage.2018.03.006.

[68] E. Kaden, N. D. Kelm, R. P. Carson, M. D. Does, D. C. Alexander, Multi-compartment microscopic diffusion imaging, NeuroImage 139 (2016) 346-359. doi:10.1016/j.neuroimage.2016.06.002.

[69] D. C. Alexander, P. L. Hubbard, M. G. Hall, E. A. Moore, M. Ptito, G. J. Parker, T. B. Dyrby, Orientationally invariant indices of axon diameter and density from diffusion MRI, NeuroImage 52 (4) (2010) 1374-1389. doi:10.1016/j.neuroimage.2010.05.043.

[70] D. Barazany, P. J. Basser, Y. Assaf, In vivo measurement of axon diameter distribution in the corpus callosum of rat brain, Brain 132 (5) (2009) 1210-1220. doi:10.1093/brain/awp042.

[71] J. D. Tournier, F. Calamante, A. Connelly, Robust determination of the fibre orientation distribution in diffusion MRI: Non-negativity constrained super-resolved spherical deconvolution, NeuroImage 35 (4) (2007) 1459-1472. doi:10.1016/j.neuroimage.2007.02.016. 
[72] V. J. Wedeen, D. L. Rosene, R. Wang, G. Dai, F. Mortazavi, P. Hagmann, J. H. Kaas, W. Y. I. Tseng, The geometric structure of the brain fiber pathways, Science 335 (6076) (2012) 1628-1634. doi:10.1126/science.1215280.

[73] M. Catani, I. Bodi, F. Dell'Acqua, Comment on "The geometric structure of the brain fiber pathways", Science 337 (6102) (2012) 1605-1605. doi:10.1126/science.1223425.

[74] V. J. Wedeen, D. L. Rosene, R. Wang, G. Dai, F. Mortazavi, P. Hagmann, J. H. Kaas, W. Y. I. Tseng, Response to comment on "The geometric structure of the brain fiber pathways" (Sep. 2012). doi:10.1126/science.1223493.

[75] J. Rafael-Patino, D. Romascano, A. Ramirez-Manzanares, E. J. Canales-Rodríguez, G. Girard, J.-P. Thiran, Robust Monte-Carlo Simulations in Diffusion-MRI: Effect of the Substrate Complexity and Parameter Choice on the Reproducibility of Results, Frontiers in Neuroinformatics 14 (2020) 8. doi:10.3389/fninf.2020.00008.

[76] R. Callaghan, D. C. Alexander, M. Palombo, H. Zhang, ConFiG: Contextual Fibre Growth to generate realistic axonal packing for diffusion MRI simulation, NeuroImage 220 (2020) 117107. doi:10.1016/j.neuroimage.2020.117107.

[77] E. Panagiotaki, M. G. Hall, H. Zhang, B. Siow, M. F. Lythgoe, D. C. Alexander, High-fidelity meshes from tissue samples for diffusion MRI simulations, in: Lecture Notes in Computer Science (including subseries Lecture Notes in Artificial Intelligence and Lecture Notes in Bioinformatics), Vol. 6362 LNCS, Springer, Berlin, Heidelberg, 2010, pp. 404-411. doi:10.1007/978-3-642-15745-5_50.

[78] G. Adluru, Y. Gur, J. S. Anderson, L. G. Richards, N. Adluru, E. V. Dibella, Assessment of white matter microstructure in stroke patients using NODDI, in: 2014 36th Annual International Conference of the IEEE Engineering in Medicine and Biology Society, EMBC 2014, Vol. 2014, Institute of Electrical and Electronics Engineers Inc., 2014, pp. 742-745. doi:10.1109/EMBC.2014.6943697.

[79] T. Taoka, N. Aida, Y. Fujii, K. Ichikawa, H. Kawai, T. Nakane, R. Ito, S. Naganawa, White matter microstructural changes in tuberous sclerosis: Evaluation by neurite orientation dispersion and density imaging (NODDI) and diffusion tensor images, Scientific Reports 10 (1) (2020) 1-9. doi:10.1038/s41598-019-57306-w.

[80] A. Hagiwara, K. Kamagata, K. Shimoji, K. Yokoyama, C. Andica, M. Hori, S. Fujita, T. Maekawa, R. Irie, T. Akashi, A. Wada, M. Suzuki, O. Abe, N. Hattori, S. Aoki, White matter abnormalities in multiple sclerosis evaluated by quantitative synthetic MRI, diffusion tensor imaging, and neurite orientation dispersion and density imaging, American Journal of Neuroradiology 40 (10) (2019) 1642-1648. doi:10.3174/ajnr.A6209.

[81] I. Timmers, A. Roebroeck, M. Bastiani, B. Jansma, E. Rubio-Gozalbo, H. Zhang, Assessing microstructural substrates of white matter abnormalities: A Comparative study using DTI and NODDI, PLoS ONE 11 (12) (2016) e0167884. doi:10.1371/journal.pone.0167884.

[82] T. Schneider, W. Brownlee, H. Zhang, O. Ciccarelli, D. H. Miller, C. G. Wheeler-Kingshott, Sensitivity of multishell NODDI to multiple sclerosis white matter changes: a pilot study, Functional Neurology 32 (2) (2017) 97. doi:10.11138/FNEUR/2017.32.2.097.

[83] K. G. Schilling, V. Janve, Y. Gao, I. Stepniewska, B. A. Landman, A. W. Anderson, Histological validation of diffusion MRI fiber orientation distributions and dispersion, NeuroImage 165 (2017) 200-221. doi:10.1016/j.neuroimage.2017.10.046.

[84] F. Grussu, T. Schneider, C. Tur, R. L. Yates, M. Tachrount, A. Ianu, M. C. Yiannakas, J. Newcombe, H. Zhang, D. C. Alexander, G. C. DeLuca, C. A. M. Gandini Wheeler-Kingshott, Neurite dispersion: a new marker of multiple sclerosis spinal cord pathology?, Annals of Clinical and Translational Neurology 4 (9) (2017) 663-679. doi:10.1002/acn3.445.

[85] C. M. Tax, F. Szczepankiewicz, M. Nilsson, D. K. Jones, The dot-compartment revealed? Diffusion MRI with ultra-strong gradients and spherical tensor encoding in the living human brain, NeuroImage 210 (2020) 116534. doi:10.1016/j.neuroimage.2020.116534. 